\title{
UICN
}

\section{Microplastiques primaires dans les océans :}

évaluation mondiale des sources

Julien Boucher, Damien Friot

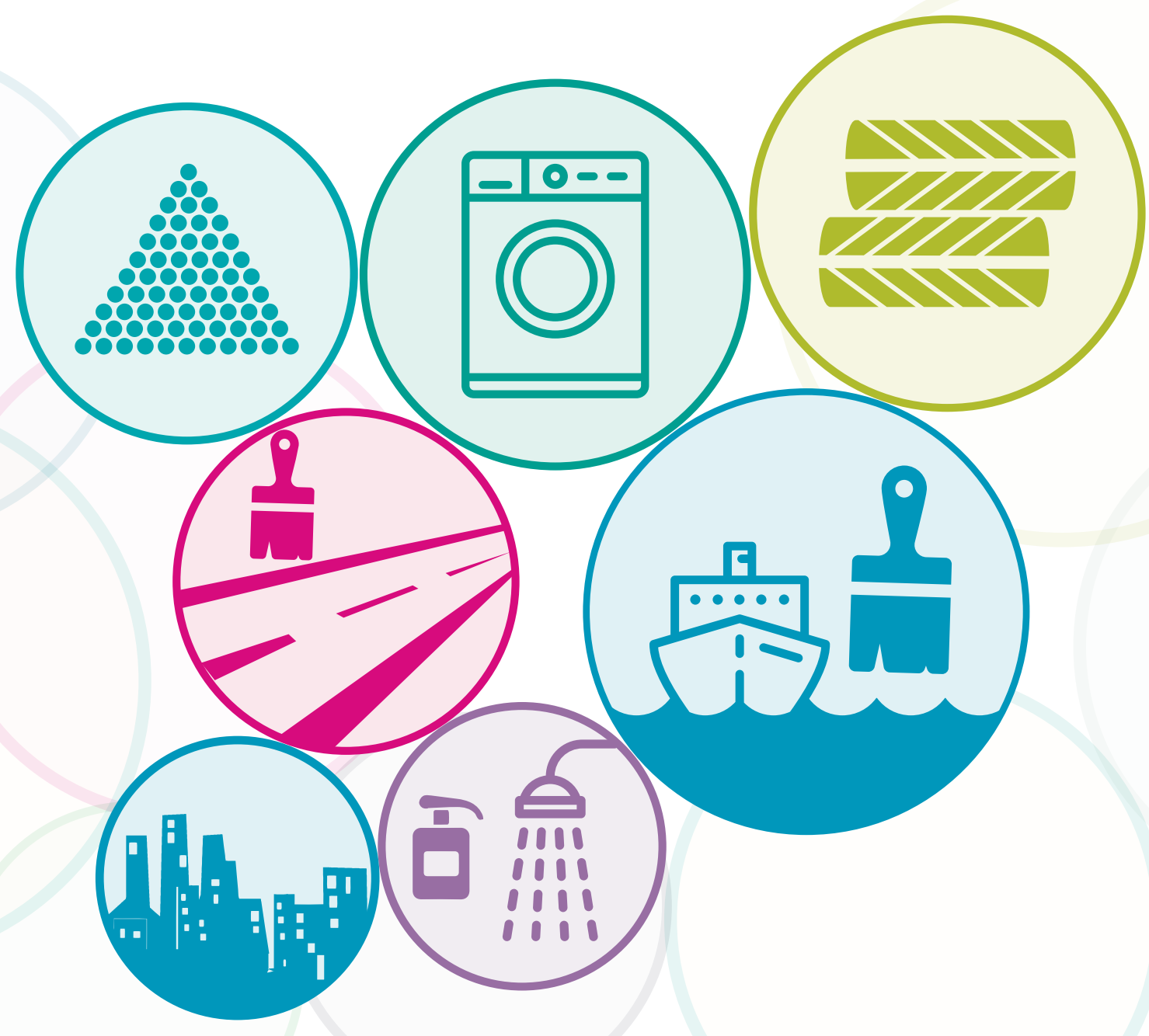




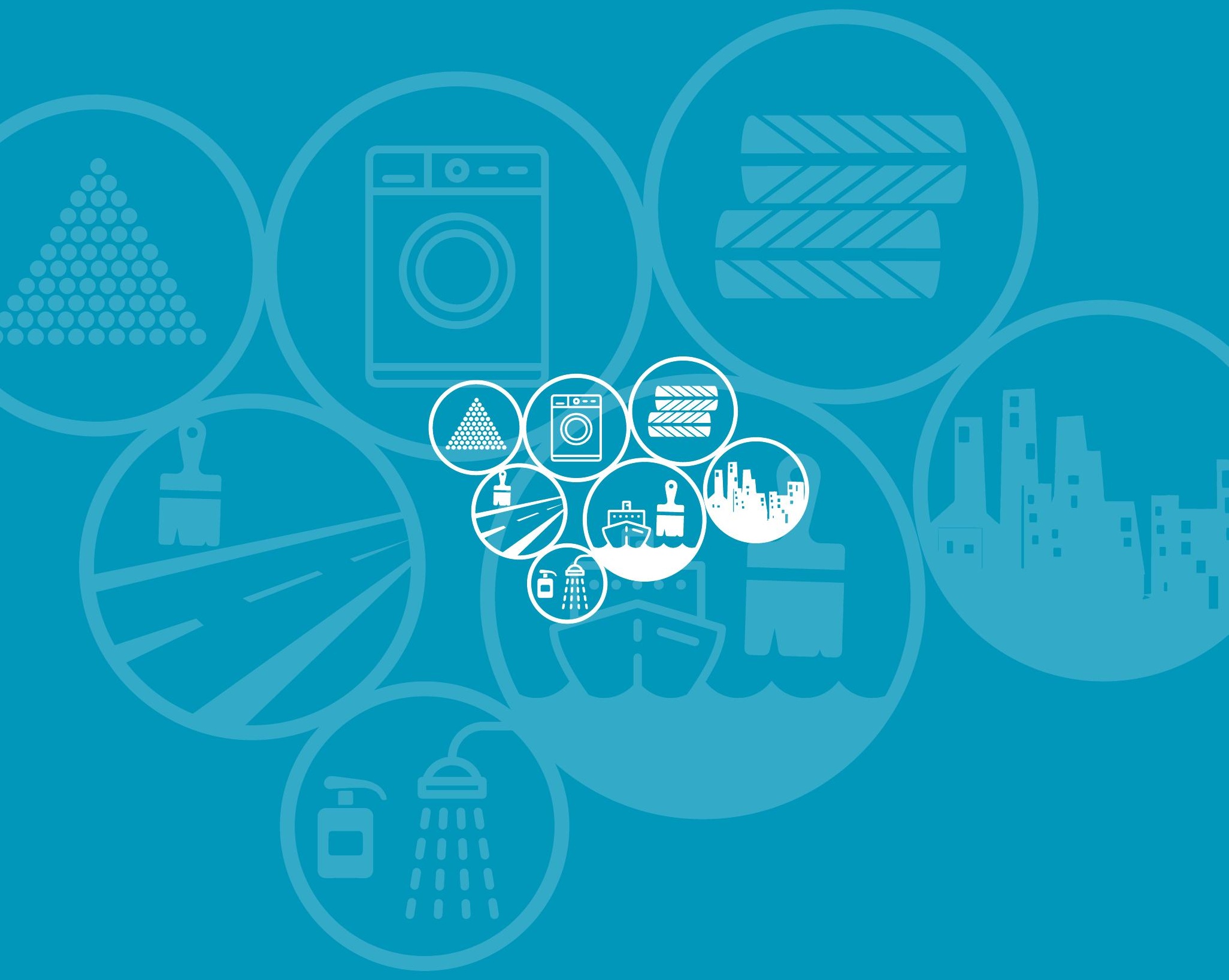




\section{Microplastiques primaires dans les océans :}

évaluation mondiale des sources

Julien Boucher, Damien Friot 
La terminologie géographique utilisée dans cet ouvrage, de même que sa présentation, ne sont en aucune manière l'expression d'une opinion quelconque de la part de I'UICN sur le statut juridique ou l'autorité de quelque pays, territoire ou région que ce soit ou sur la délimitation de ses frontières ou limites.

Les opinions exprimées dans la présente publication ne reflètent pas nécessairement celles de l'UICN.

L'UICN rejette toute responsabilité en cas d'erreurs ou d'omissions intervenues lors de la traduction en français de ce document dont la version originale est en anglais. En cas de divergences, veuillez vous référer à l'édition originale. Titre de l'édition originale : Primary microplastics in the oceans: a global evolution of sources. (2017). DOI: https://doi.org/10.2305/IUCN.CH.2017.01.en

Publié par: $\quad$ UICN, Gland, Suisse

Droits d'auteur: $\quad$ (c) $2020 \mathrm{UICN}$, Union internationale pour la conservation de la nature et de ses ressources

La reproduction de cette publication à des fins non commerciales, notamment éducatives, est permise sans autorisation préalable écrite du détenteur des droits d'auteur à condition que la source soit dûment citée.

La reproduction de cette publication à des fins commerciales, notamment en vue de la vente, est interdite sans autorisation préalable écrite du détenteur des droits d'auteur.

Citation: $\quad$ Boucher, J. et Friot D. (2020). Microplastiques primaires dans les océans : évaluation mondiale des sources. Gland, Suisse : UICN. 44 p.

ISBN :

$978-2-8317-2022-7$

DOI :

https://doi.org/10.2305/IUCN.CH.2017.01.fr

Traduction : Hélène Tagand

Auteurs: $\quad$ Julien Boucher

EA - Shaping Environmental Action \& Haute École spécialisée de Suisse occidentale // HES-SO, HEIG-VD, Yverdon-les-Bains, Suisse

Damien Friot

EA - Shaping Environmental Action

www.shaping-ea.com

contact@shaping-ea.com

Relecteurs: $\quad$ Carl Gustaf Lundin et João Matos de Sousa

Relecteurs externes: Francis Vorhies (Earthmind, http://earthmind.org)

François Galgani (IFREMER, Laboratoire LER/PAC)

Mathieu Pernice (University of Technology, Sydney)

Doug Woodring (www.oceanrecov.org)

Mise en page : Imre Sebestyén jr. (Unit Graphics Studio)

Disponible auprès de : www.iucn.org/resources/publications 


\section{SOMMAIRE}

Avant-propos.

1. Résumé

2. Introduction. . . . . . . . . . . . . . . . . . . . . . . . . . . . . . .

2.1 Plastiques et microplastiques polluent les océans du monde. . . . . . . . . . . . . . . . 8

2.2 Rejets de microplastiques : des connaissances lacunaires. . . . . . . . . . . . . . . . . . . 9

2.3 Une consommation de plastique en plein essor . . . . . . . . . . . . . . . . . . . . . 10

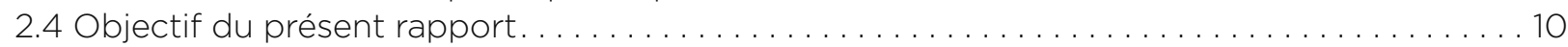

3. Description des enjeux et méthodologie. . . . . . . . . . . . . . . . . . . . . 11

3.1 Principales sources identifiées dans la littérature scientifique $\ldots \ldots \ldots \ldots \ldots$

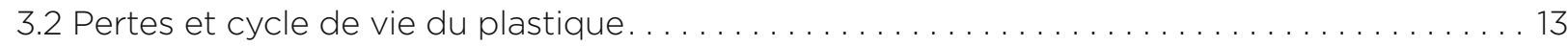

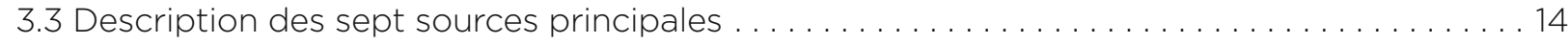

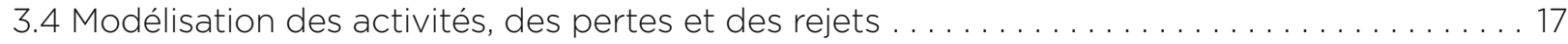

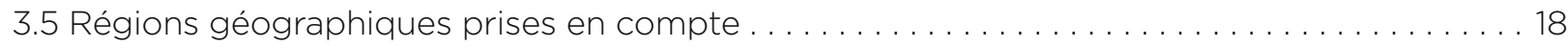

3.6 Présentation des résultats selon trois scénarios $\ldots \ldots \ldots \ldots \ldots \ldots \ldots \ldots \ldots$

4. Résultats et analyse ...................................... 19

4.1 Les microplastiques primaires, une part substantielle du plastique des océans . . . . . . . . 20

4.2 L'usure de textiles et des pneus à l'origine de deux tiers des rejets . . . . . . . . . . . . . . 21

4.3 Trois quarts des rejets domestiques dus à l'utilisation et l'entretien des produits . . . . . . 23

4.4 Deux tiers des pertes dues aux eaux de ruissellement des routes . . . . . . . . . . . . . . 24

4.5 Une contribution significative de toutes les régions aux rejets . . . . . . . . . . . . . . . . . . . . . .

4.6 Rejets moyens : un sac en plastique par personne et par semaine . . . . . . . . . . . . . . 26

4.7 Principales sources de rejets par région . . . . . . . . . . . . . . . . . . . . . . . 27

4.8 Rejets de microplastiques élevés en Europe et en Amérique du Nord. . . . . . . . . . . . . . . . . 28

4.9 Nécessité de varier les solutions pour stopper le flot de plastique . . . . . . . . . . . . . . . . . 29

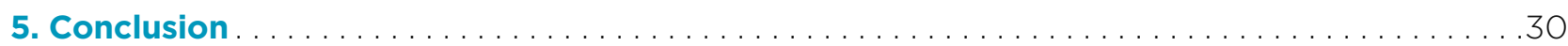

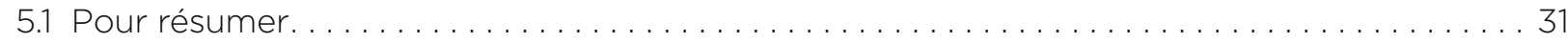

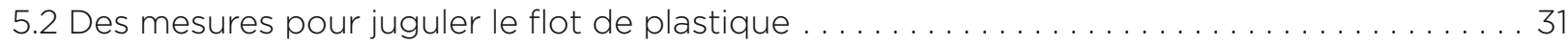

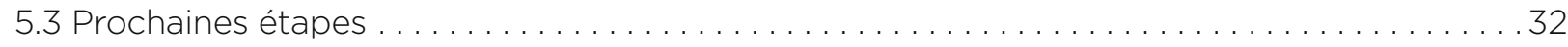

6. Annexe 1 : Sources . . . . . . . . . . . . . . . . . . . . . . . . . . . 33

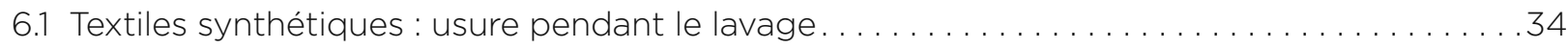

6.2 Pneus : abrasion sur la route . . . . . . . . . . . . . . . . . . . . . . . . . 34

6.3 Poussières urbaines : déversements, exposition aux intempéries et abrasion. . . . . . . . . 35

6.4 Marquage routier : exposition aux intempéries et abrasion par les véhicules . . . . . . . . . . 36

6.5 Peintures marines : exposition aux intempéries, application et entretien. . . . . . . . . . 36

6.6 Articles d'hygiène et de soin : pertes pendant l'utilisation . . . . . . . . . . . . . . . 37

6.7 Granulés de plastique : fabrication, transport et recyclage . . . . . . . . . . . . . 37

7. Annexe 2 : Parcours des plastiques . . . . . . . . . . . . . . . . . . . . 38

7.1 Parcours des eaux de ruissellement des infrastructures routières . . . . . . . . . . . . . . 39

7.2 Parcours des eaux usées. . . . . . . . . . . . . . . . . . . . . . . . . . . . . 39

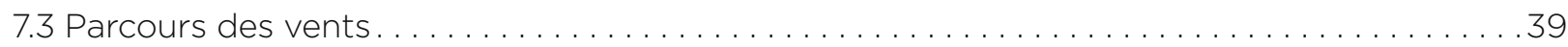

7.4 Parcours des océans . . . . . . . . . . . . . . . . . . . . . . . . . . . . . . 39

8. Annexe 3 : Régions étudiées. . . . . . . . . . . . . . . . . . . . . 40

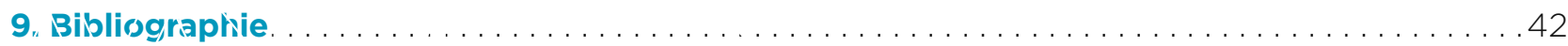




\section{Avant-propos}

L'invention du plastique à partir d'un polymère de synthèse en 1907 a bouleversé nos vies à jamais, pour le meilleur et pour le pire. Le plastique est l'un des matériaux les plus polyvalents jamais produits. II a révolutionné notre manière d'emballer, de manger, de voyager et de nous habiller.

La révolution du plastique a toutefois un prix. Le plastique pollue notre environnement marin à une échelle alarmante : 9,5 millions de tonnes de nouveaux plastiques se déversent chaque année dans l'océan. $\mathrm{Ce}$ plastique détruit notre précieuse biodiversité et les fragiles écosystèmes dont nous dépendons. La pollution de nos océans est également en train de devenir une menace pour la santé humaine, le plastique s'insinuant dans nos ressources alimentaires et hydriques.

En dépit d'un volume croissant d'études sur les débris de plastique et une sensibilisation accrue aux conséquences de leur accumulation au niveau mondial, les efforts entrepris pour combattre cette pollution ont été contrariés par le manque de recherches et de connaissances sur les sources originelles de cette matière résiduelle.

Le rapport Microplastiques primaires dans les océans : évaluation mondiale des sources contribue à combler cette lacune et fournit aux décideurs un point de référence important pour les aider à élaborer et à mettre en œuvre les politiques transformatrices et les nouvelles pratiques de production nécessaires pour passer à une économie mondiale circulaire.

Dans la droite ligne du programme Close the plastic tap et de la publication Plastic debris in the ocean de I'UICN, ce rapport fournit une estimation et une cartographie mondiales des sources et des quantités de microplastiques primaires, c'est-à-dire ceux qui pénètrent dans l'océan sous la forme de petites particules issues de produits domestiques et industriels.
Bien que la mauvaise gestion des déchets en plastique demeure la principale source de la pollution marine par les plastiques dans le monde, ce rapport montre pour la première fois que dans certains pays, il se pourrait que l'utilisation de véhicules et le lavage du linge contribuent davantage à la pollution par le plastique que la mauvaise gestion de nos déchets.

Faire face aux multiples sources de plastiques marins suppose d'adopter une approche holistique qui s'attaque au problème à sa racine. Les observations décrites dans ce rapport doivent susciter une nouvelle réflexion sur notre manière de concevoir, produire, consommer et éliminer les plastiques. Tarir le flot de plastique pourrait supposer de nouveaux matériaux et une conception judicieuse, comme des vêtements qui perdent moins de fibres ou des machines à laver pourvues de meilleurs filtres. Ces efforts doivent être soutenus par une législation et des politiques concrètes qui forcent un réel changement.

Ce rapport nous rappelle également qu'en tant que consommateurs de plastiques, il nous incombe de nous informer et d'adapter notre comportement pour préserver notre planète bleue.

La tâche à accomplir est immense, mais par nos efforts conjugués nous pouvons déjouer les sombres prévisions concernant la pollution par le plastique et rendre la santé à nos océans.

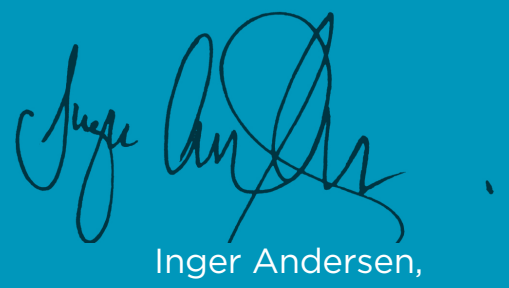

Directeur général de l'UICN

Le présent rapport doit son existence au précieux soutien de MAVA, Fondation pour la Nature. Nous remercions également la Swedish Postcode Foundation et la Gallifrey Foundation pour leur appui au lancement de cette évaluation. 


\section{Résumé}

Le plastique a pénétré chaque aspect de notre vie : des vêtements aux peintures en passant par les véhicules et les produits d'hygiène. Le plastique est bon marché, durable, léger et malléable, ses usages sont donc quasi illimités. Les inconvénients du plastique deviennent cependant de plus en plus évidents : il fuit massivement dans les rivières et les océans, avec des effets dévastateurs sur les écosystèmes marins et les activités économiques qui en dépendent.

Les déchets de plastique se composent de résidus de toutes tailles, des gros objets facilement retirables aux particules invisibles à l'œil nu. Le présent rapport étudie les sources des microplastiques primaires, c'est-à-dire les particules de plastique d'une taille inférieure à $5 \mathrm{~mm}$ qui se retrouvent directement dans l'environnement. Ils se distinguent des microplastiques secondaires, qui proviennent principalement de la dégradation, due à leur exposition à l'environnement marin, de déchets en plastique plus volumineux. Les microplastiques primaires peuvent être volontairement ajoutés à des produits, comme certains agents exfoliants incorporés dans des cosmétiques (gels douche, crèmes, etc.) ou ils peuvent résulter de l'abrasion d'objets en plastique plus gros, au cours de leur fabrication, de leur utilisation ou de leur entretien, comme l'usure des pneus sur les routes ou celle des textiles synthétiques pendant le lavage.

Le présent rapport est l'un des premiers de ce type à quantifier les fuites de microplastiques primaires et à montrer qu'elles constituent l'un des principaux facteurs de la présence de plastique dans les océans. Le modèle développé pour cette analyse nous permet de conclure qu'entre 15 et 31 \% de tout le plastique présent dans les océans pourraient être primaires. Cette proportion considérable est encore largement ignorée. Dans certains pays équipés de systèmes de traitement des déchets perfectionnés, les rejets de microplastiques primaires dépassent même ceux de microplastiques secondaires.

Les rejets mondiaux de microplastiques primaires dans les océans ont été estimés à 1,5 million de tonnes par an (Mtonnes/an), avec des estimations comprises entre 0,8 et 2,5 Mtonnes/an selon que ces scénarios sont optimistes ou pessimistes. Ce chiffre mondial correspond à 212 grammes par personne, soit l'équivalent, dans le monde, d'un sac d'épicerie vide jeté dans l'océan chaque semaine par chaque habitant.

L'écrasante majorité des microplastiques primaires rejetés (98\%) sont issus d'activités terrestres. Seuls $2 \%$ sont produits par des activités qui se déroulent en mer. La majeure partie de ces particules proviennent du lavage de textiles synthétiques et de l'abrasion des pneus sur les routes. L'utilisation des produits contribue aux rejets dans les océans à hauteur de $49 \%$ et l'entretien des produits à hauteur de $28 \%$. Ces microplastiques sont acheminés dans l'océan par le ruissellement de l'eau sur les routes (66\%), les systèmes de traitement des eaux usées (25\%) et le vent (7\%).

Cette étude porte sur sept régions : l'Afrique et le Moyen-Orient, la Chine, l'Asie de l'Est et l'Océanie, l'Europe et l'Asie centrale, l'Inde et l'Asie du Sud, l'Amérique du Nord et l'Amérique du Sud. Elle a révélé des rejets par région comparables en valeur absolue (s'échelonnant de 134 à 281 milliers de tonnes/an). Les rejets par personne sont cependant très hétérogènes et s'échelonnent entre 110 et 750 grammes/personne/an. De plus, les prévisions font état d'un accroissement des rejets de microplastiques primaires dans la plupart des régions au cours des prochaines décennies, en raison de l'augmentation escomptée du revenu par personne, si rien n'est fait pour améliorer les systèmes permettant d'éviter ces rejets.

II importe de noter que le présent rapport se fonde principalement sur des modélisations et des informations relatives aux fuites provenant d'activités économiques et domestiques et ne repose que sur des données 
publiques et non sur des mesures effectuées sur le terrain. Le modèle pourrait être encore perfectionné par l'utilisation de données propriétaires payantes sur les quantités de plastique par région. II pourrait en outre être renforcé en affinant les hypothèses comportementales régionales de base. II conviendrait enfin, pour valider ce modèle prédictif, de le confronter aux données empiriques de terrain. Cette confrontation est toutefois impossible pour l'heure, compte tenu du manque d'études et de l'absence de protocole expérimental adéquat pour réaliser cette comparaison. L'éventail de scénarios pessimistes et optimistes envisagés tout au long de l'étude nous laisse néanmoins penser que les ordres de grandeur que nous avançons sont fiables.

Ce rapport met en lumière la part des microplastiques primaires dans les océans à l'échelle mondiale. II ouvre la voie à un nouveau répertoire d'actions de lutte contre les rejets de plastiques dans les océans qui dépasse le champ traditionnel de la gestion des déchets. Penser ces solutions supposera de réfléchir en termes d'écoconception des produits, de cycle de vie et d'implication des acteurs clés du secteur privé (industries textile et automobile) comme du secteur public (traitement des eaux et aménagement du territoire). 


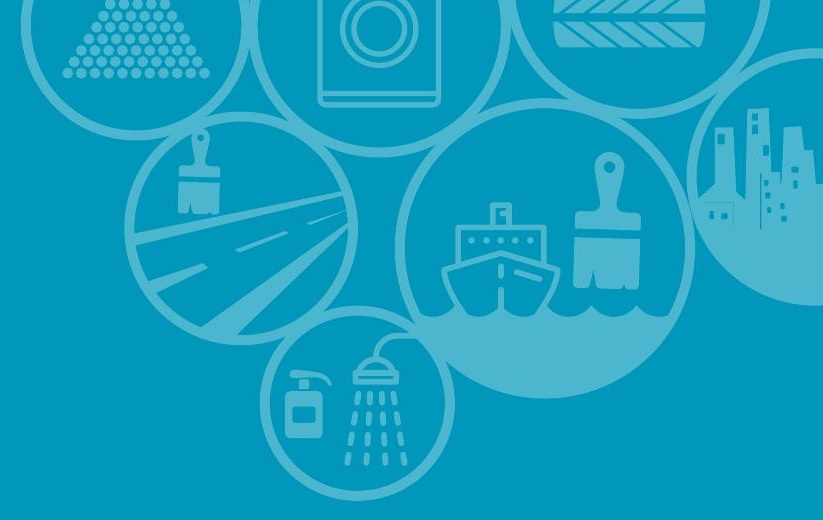

Le plastique a pénétré chaque aspect de notre vie: des vêtements aux peintures en passant par les véhicules et les produits d'hygiène. Le plastique est bon marché, durable, léger et malléable, ses possibilités sont donc quasi illimitées.

Les inconvénients du plastique deviennent cependant de plus en plus évidents : il fuit massivement dans les rivières et les océans, avec des effets dévastateurs sur les écosystèmes marins et les activités économiques qui en dépendent.

On entend souvent que la plupart des plastiques qui se retrouvent dans l'océan proviennent de déchets abandonnés dans la nature ou d'une mauvaise prise en charge des déchets. Or, bien que ne faisant pas les gros titres, les microplastiques primaires sont en train de devenir une source de préoccupation majeure. Leur propagation est bien plus discrète, elle résulte de l'ajout volontaire de microbilles dans des produits comme des cosmétiques ou de l'usure d'objets en plastique plus gros comme des textiles ou des pneus.

L'objet de ce rapport est de fournir les premières estimation et cartographie mondiales des sources de microplastiques et des quantités rejetées dans l'océan. Il vise à donner aux décideurs et aux acteurs concernés de nouvelles informations afin de les aider à prendre des mesures efficaces pour juguler ce torrent de plastique.

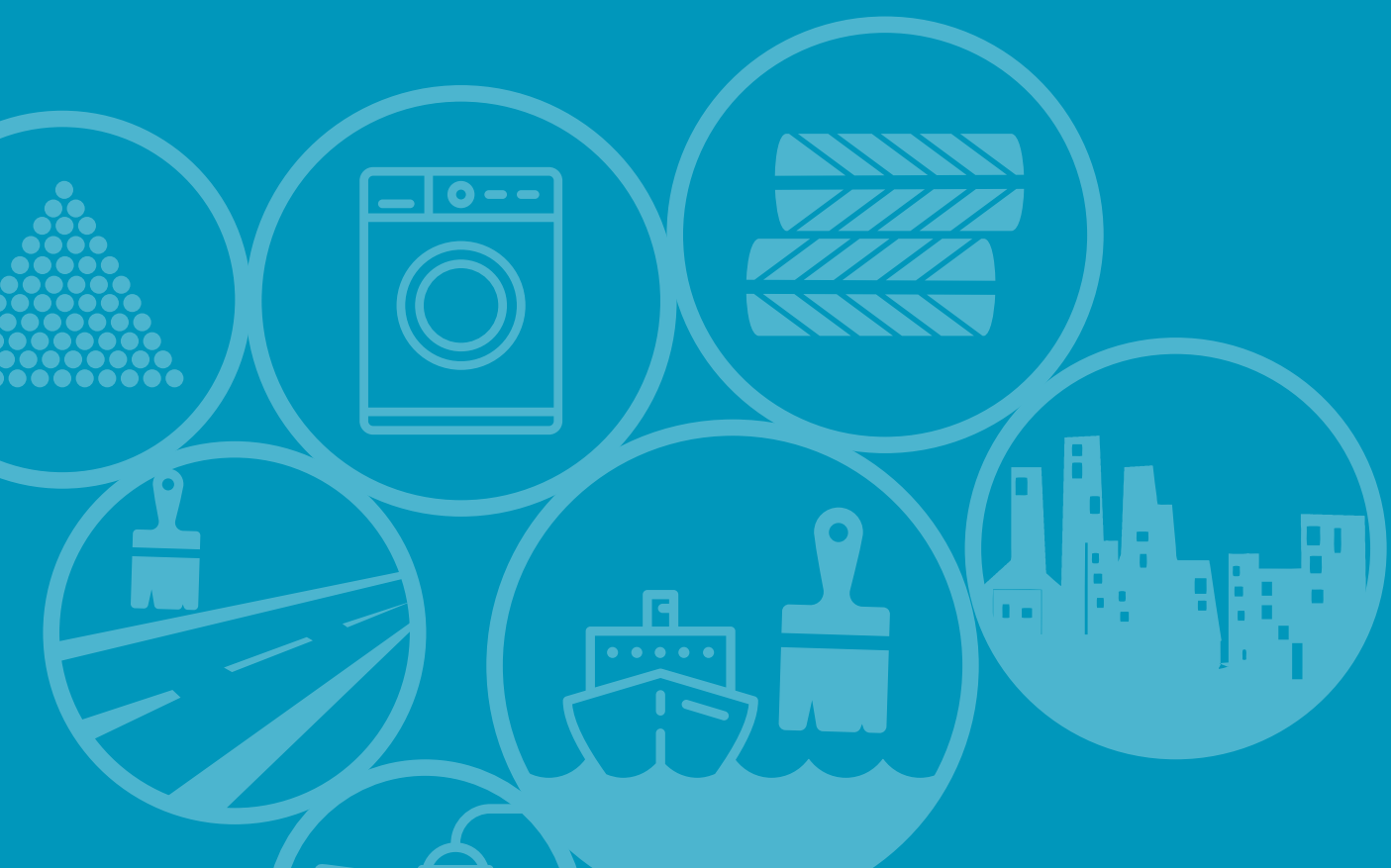




\subsection{Plastiques et microplastiques polluent les océans du monde}

Les environnements marins du monde entier sont pollués par les plastiques (GESAMP, 2015), que l'on trouve sous deux formes: les gros déchets en plastique et les particules de taille inférieure à $5 \mathrm{~mm}$, que l'on appelle les microplastiques (Thevenon et al., 2014).

De récentes études évaluent la quantité totale de plastique produite depuis son invention à 8,3 milliards de tonnes (Geyer et al., 2017). Sur ce total, près de 9 \% auraient été recyclés (Geyer et al., 2017), alors qu'on estime la quantité de plastique qui pénètre dans l'océan chaque année entre 4,8 et 12,7 millions de tonnes (Jambeck et al., 2015). Pour pouvoir citer précisément ces récentes estimations et tentatives de quantifier le problème, il est essentiel de comprendre le rapport entre macro et microplastiques.

La fourchette de 4,8 à 12,7 millions de tonnes de plastique arrivant dans l'océan chaque année ne concerne que les macroplastiques (Jambeck et al., 2015). Cette estimation est fondée sur la masse de déchets générée par personne chaque année, le pourcentage de déchets en plastique que cela représente et enfin la part de ce pourcentage qui n'est pas traitée convenablement et qui pourrait venir grossir la pollution des océans. Ainsi, les chiffres avancés dans le présent rapport sont à ajouter aux estimations figurant dans le rapport de Jambeck, ce qui porte la quantité totale de plastiques pénétrant dans l'océan, macro et micro compris, à un chiffre bien supérieur à la moyenne de 8 millions de tonnes par an communément citée.

Les gros déchets en plastique sont visibles. Des études ont déjà démontré leurs conséquences néfastes sur les plans écologique, social et économique, qui vont des empoisonnements, étouffements, lésions et enchevêtrements de la faune aux difficultés économiques du secteur maritime et touristique (GESAMP, 2015; Raynaud, 2014). Pour un panorama des effets délétères des plastiques sur les écosystèmes, voir l'étude de Thevenon et al. (2014).

Les microplastiques ne sont pas aisément visibles à l'œil nu et leurs impacts négatifs sont moins évidents, mais leur rejet dans les océans pourrait également être lourd de conséquences. L'accumulation de microplastiques dans la chaîne alimentaire et la sorption de produits toxiques par le plastique pendant son parcours dans l'environnement sont soupçonnées d'être à l'origine de troubles de la santé humaine (Eriksen et al., 2014).

Deux types de microplastiques contaminent les océans du monde : les microplastiques primaires et secondaires. La littérature scientifique en propose différentes définitions (Lassen et al., 2015). Nous avons opté pour les suivantes, issues d'une étude norvégienne (Sundt et al., 2014).

- Les microplastiques primaires sont les plastiques qui sont rejetés directement dans l'environnement sous la forme de petites particules. Ils peuvent avoir été volontairement ajoutés à des produits, comme les agents exfoliants que l'on trouve dans les articles de toilette et les cosmétiques (par exemple les gels douche). Il peuvent aussi provenir de l'usure d'objets en plastique plus gros au cours de leur fabrication, de leur utilisation ou de leur entretien, comme l'abrasion des pneus sur les routes ou le frottement des textiles synthétiques pendant le lavage.

- Les microplastiques secondaires sont les microplastiques issus de la fragmentation d'éléments en plastique plus gros exposés à l'environnement marin, notamment par photodégradation et autres effets de l'exposition aux intempéries de déchets abandonnés dans la nature, comme des sacs en plastique, ou perdus, comme des filets de pêche. L'origine des microplastiques secondaires étant difficile à établir du fait de leur désagrégation, il est malaisé d'évaluer convenablement la proportion de macroplastiques qui se sont désormais délités en microplastiques. C'est pourquoi le présent rapport porte sur la quantification des microplastiques primaires, plus réalisable avec les données dont nous disposons.

Une fois parvenus dans l'océan, les microplastiques flottent ou coulent. Ceux qui sont plus légers que l'eau de mer, comme le polypropylène, flottent et se dispersent sur de très grandes distances au gré des courants pour finalement s'accumuler dans les gyres. Eriksen et al. (2014) et Sebille et al. (2015) estiment qu'entre 93 et 268 milliers de tonnes de ces microplastiques flottent actuellement dans les océans. D'autres microplastiques comme l'acrylique sont plus denses que l'eau de mer et s'amoncellent probablement sur le plancher océanique, ce qui signifie qu'une quantité significative de microplastiques s'accumulent en haute mer (Woodall et al., 2014) et en fin de compte dans les chaînes alimentaires (Seltenrich, 2015). 
Cette pollution due aux microplastiques omniprésente dans les océans devient une grave préoccupation. En raison de l'ampleur et de la singularité de cette dégradation, certains ont dénommé la période actuelle le plasticène (Reed, 2016) et décrivent l'océan comme une soupe de plastique?.

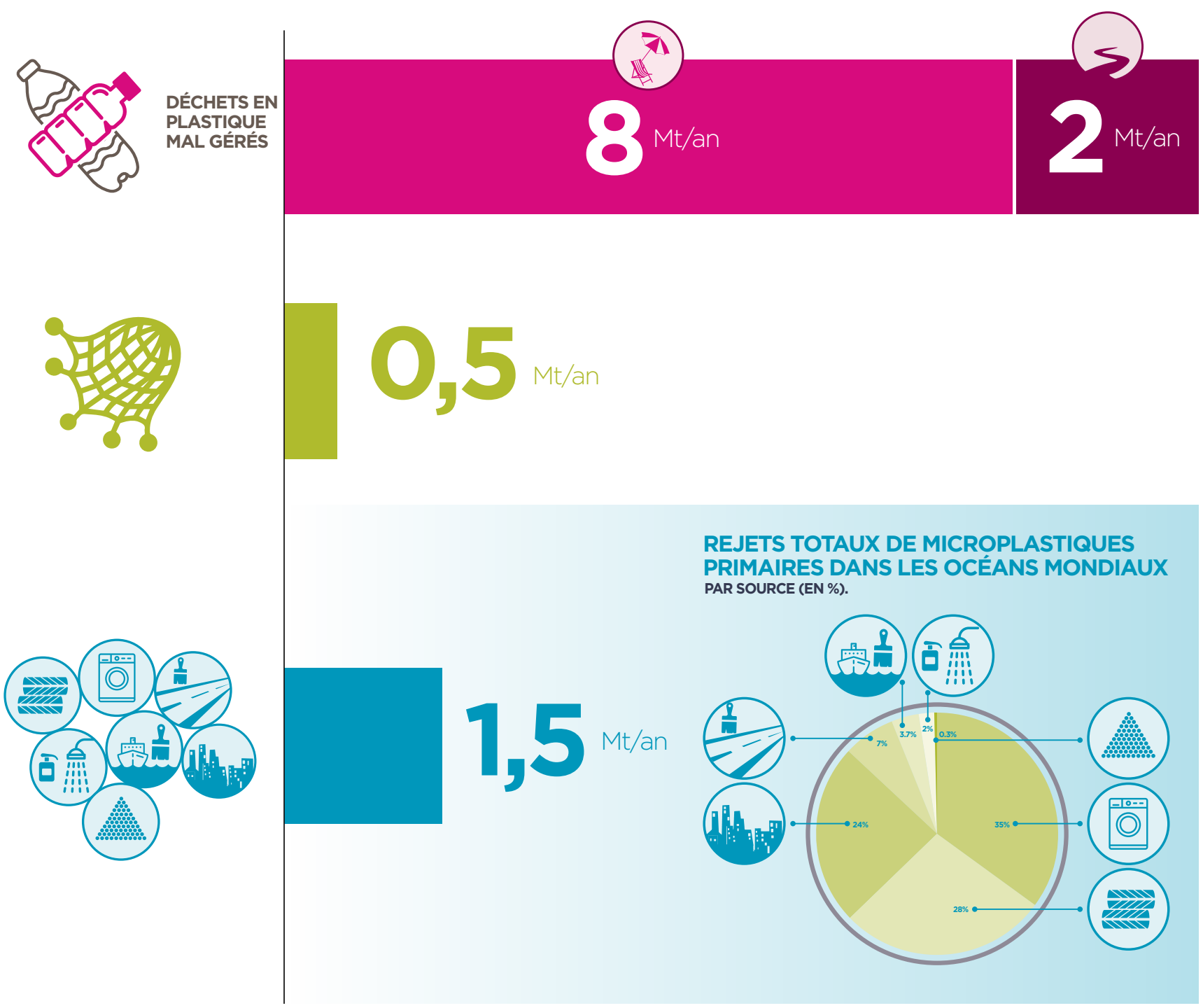

\subsection{Rejets de microplastiques : des connaissances lacunaires}

Il est communément admis que la plupart des plastiques qui se retrouvent dans l'océan proviennent de déchets mal pris en charge. La majeure partie des rapports publiés récemment s'attachent presque exclusivement à la quantification de ces sources secondaires ainsi qu'à la réduction et à la gestion des déchets (EUNOMIA, 2016 a; Jambeck et al., 2015; McKinsey \& Company and Ocean Conservancy, 2015; World Economic Forum et al., 2016). Jambeck (2015) fait état de 4,8 à 12,7 millions de tonnes de plastique rejetées dans les océans chaque année en raison d'un mauvais traitement des déchets.

Les connaissances relatives au déversement de microplastiques primaires dans les océans sont cependant lacunaires : bien que de nombreuses sources de microplastiques aient été identifiées par les scientifiques, les quantités globales rejetées n'ont pas été évaluées. Par conséquent, l'importance relative des sources primaires de microplastiques par rapport aux sources secondaires reste largement inconnue et les débats autour du plastique se cantonnent généralement à la gestion des déchets.

1 Voir: http://www.plasticsoupfoundation.org 
Les niveaux élevés de microplastiques découverts dans les lacs et rivières d'Europe, d'Amérique du Nord et d'Asie (Eerkes-Medrano et al., 2015) pourraient indiquer que les sources primaires comptent pour une part substantielle des rejets de microplastiques dans les océans. Une étude a même montré que dans le Danube autrichien, le plastique était plus abondant que les larves de poissons, principalement sous la forme de matières premières industrielles, telles que des granulés et des paillettes (Lechner et al., 2014). Deux autres études ont montré que la plupart du plastique découvert dans les eaux de surface provenait de cosmétiques comme des nettoyants pour le visage ou de textiles (Browne et al., 2011; Driedger et al., 2015). Dans les récentes évaluations réalisées à l'échelle d'un pays en Europe, Essel et al. (2015), Lassen et al. (2015), Magnuson et al. (2016), RIVM (2014), Sundt et al. (2014) ont identifié et quantifié près de quinze sources de microplastiques. Les pneus sont généralement cités comme étant la source principale.

\subsection{Une consommation de plastique en plein essor}

Le plastique est aujourd'hui un matériau si commun qu'il fait partie intégrante de notre vie quotidienne: conditionnement, construction, y compris d'immeubles, véhicules, appareils électriques et électroniques, production agricole, vêtements et chaussures, articles de toilette et cosmétiques. À la fois durable, malléable, léger et bon marché, le plastique s'est imposé pratiquement partout.

L'utilisation du plastique a augmenté de manière exponentielle depuis que les premiers polymères organiques de synthèse ont été mis au point au milieu du 20e siècle. Plus de 300 millions de tonnes de plastique sont actuellement produites chaque année pour fabriquer des objets, un chiffre à comparer au 1,5 million de tonnes produites en 1950. Le taux de croissance annuel moyen de la production de plastique s'élève à environ $4 \%$ (PlasticsEurope, 2015). À ces chiffres s'ajoutent les matières plastiques prévues pour d'autres usages, qui ne sont pas prises en compte dans ces statistiques ${ }^{2}$, comme les fibres synthétiques destinées à l'industrie textile (37,2 millions de tonnes) ou le caoutchouc synthétique utilisé dans les pneus (6,4 millions de tonnes) 3 . De plus, selon PlasticsEurope (2015), près de $4 \%$ de la production mondiale annuelle de pétrole sert à la fabrication de plastique, alors qu'un pourcentage similaire sert à fournir l'énergie nécessaire à ce processus.

Le recours au plastique varie amplement d'un continent à l'autre. En Amérique du Nord et en Europe, par exemple, la consommation d'objets en plastique par personne s'élevait approximativement à $100 \mathrm{~kg}$ par an en 2005 et selon les prévisions elle devait atteindre $140 \mathrm{~kg}$ en 2015. Dans les pays asiatiques, la consommation individuelle d'objets en plastique est bien plus faible, $20 \mathrm{~kg}$ par personne et par an en 2005 et $36 \mathrm{~kg}$ escomptés en 2015. Elle est encore plus faible en Afrique: environ $16 \mathrm{~kg}$ par personne en 2015, selon les estimations (PlasticsEurope, 2009).

En raison des excellentes propriétés fonctionnelles de ce matériau, il paraît clair que son utilisation va encore s'intensifier, notamment dans les régions à faible revenu qui vont voir leurs économies croitre. II convient donc de trouver des solutions pour tarir le flot de plastique et réduire les effets néfastes de son utilisation sur l'environnement et potentiellement la santé humaine.

\subsection{Objectif du présent rapport}

L'objectif du présent rapport est de fournir l'une des premières évaluations quantitatives mondiales des rejets directs de microplastiques primaires d'origine pétrochimique dans les océans. Ce rapport entend contribuer à de meilleures identification et hiérarchisation des sources et des itinéraires de la pollution par les microplastiques, aux fins de permettre aux décideurs et aux principaux acteurs concernés de prendre des décisions informées et des mesures ciblées pour résoudre le problème.

2 Les utilisations autres que la fabrication d'objets ne sont pas prises en compte par les associations de fabricants de plastique (Plastics Europe, communication personnelle).

3 Les références se trouvent dans l'annexe méthodologique. 


\subsection{Principales sources identifiées dans la littérature scientifique}

De nombreuses activités, commerciales ou domestiques, sur terre ou sur mer, emploient des plastiques. Les principales sources de microplastiques primaires connues sont répertoriées et classées dans le tableau 1, en fonction de données récemment publiées au Danemark (Lassen et al., 2015), en Suède (Magnuson et al., 2016), en Norvège (Sundt et al., 2014), et en Allemagne (Essel et al., 2015).

Dans notre évaluation, les pertes et les rejets de microplastiques primaires sont quantifiés à l'échelle mondiale pour les sept sources les plus importantes identifiées par les études précédemment citées, qui sont les suivantes:

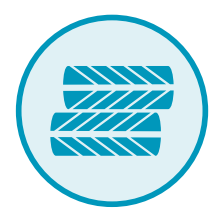

PNEUS

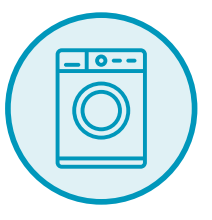

TEXTILES SYNTHETIQUES

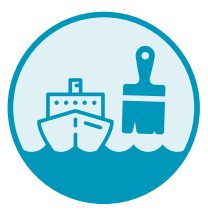

PEINTURES MARINES

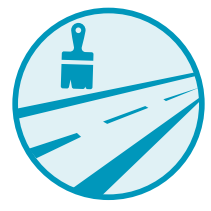

MARQUAGE
ROUTIER

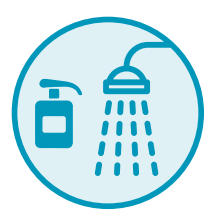

ARTICLES DE TOILETTE

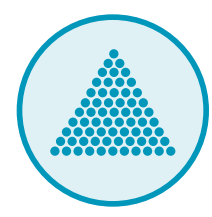

GRANULÉS DE PLASTIQUE

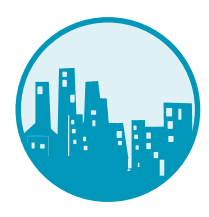

POUSSIÈRES URBAINES

Ces sources relèvent de différentes activités commerciales ou domestiques, sur terre comme sur mer, répertoriées dans le tableau 1.

Tableau 1

\section{PRINCIPALES SOURCES DE MICROPLASTIQUES}

\section{IDENTIFIÉES PAR LES ÉTUDES SCIENTIFIQUES}

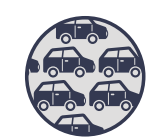

TRANSPORT DE
PASSAGERS

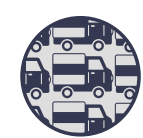

TRANSPORT DE

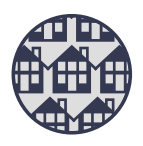

ACTIVITÉS

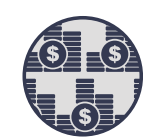

ACTIVITÉS
COMMERCIALES

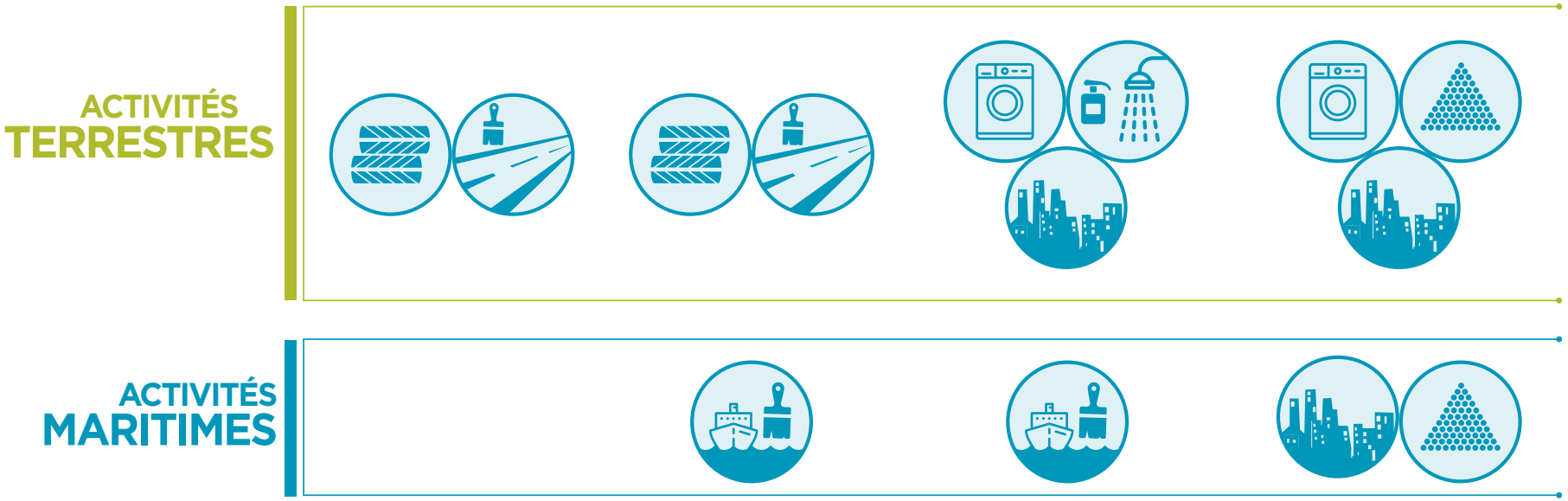




\subsection{Pertes et cycle de vie du plastique}

Les pertes de microplastiques primaires ont lieu à différentes étapes du cycle de vie du plastique et de l'objet en plastique (figure 1). Les pertes de granulés de plastique sont les seules qui peuvent survenir à la fois pendant la production, le transport et le recyclage du plastique. À la différence des microplastiques secondaires, qui résultent de la mauvaise gestion des déchets au moment de l'élimination des produits contenant du plastique, la plupart des pertes surviennent pendant l'utilisation de l'objet contenant du plastique (comme la conduite d’un véhicule) ou son entretien (comme le lavage de vêtements).

\section{Figure 1}

\section{CYCLE DE VIE DU PLASTIQUE ET DES OBJETS EN PLASTIQUE}

\section{INDIQUANT LES SOURCES DES PERTES DE} MICROPLASTIQUES PRIMAIRES

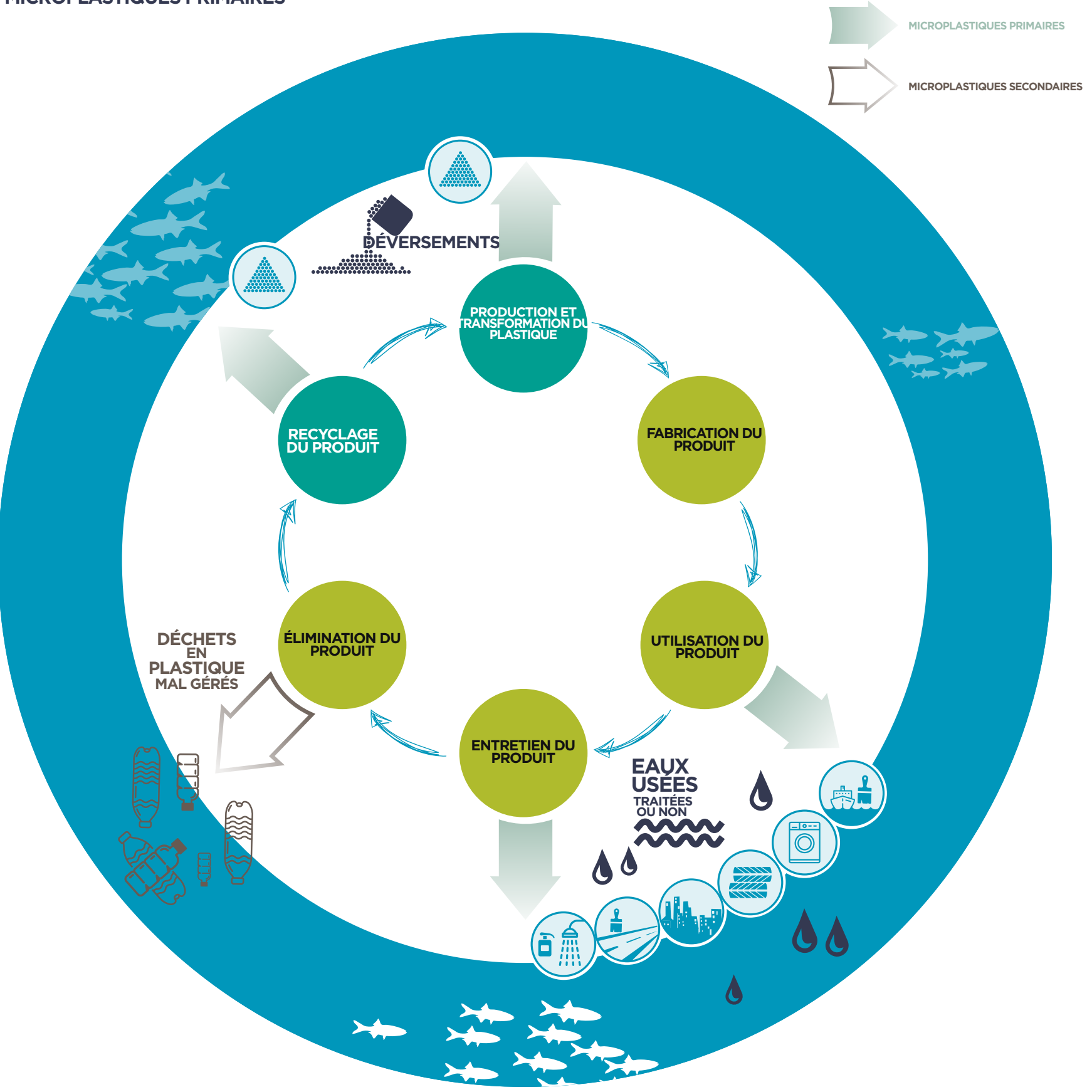




\subsection{Description des sept sources principales}

Les sources de microplastiques étudiées dans ce rapport ressortissent à une consommation mondiale de plastique qui dépasse de peu les 300 millions de tonnes, comme le résume le tableau 2. En général, ce plastique prend d'abord la forme de granulés (85\%), qui serviront à fabriquer des textiles synthétiques (12 \%) et du

caoutchouc synthétique employé dans les pneus (2\%).

Les pertes issues des articles de toilette et des cosmétiques sont les seules qui peuvent être considérées comme volontaires, car il s'agit de produits contenant des microplastiques qui sont intentionnellement versés dans l'eau. En revanche, les autres sources génèrent des pertes involontaires, qui sont la conséquence de l'abrasion, de l'exposition aux intempéries ou du déversement accidentel pendant la production, le transport, l'utilisation, l'entretien ou le recyclage des objets contenant du plastique.

\section{SOURCES ÉTUDIÉES}

\section{SOURCES}

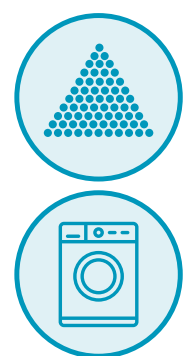

TEXTILES

SYNTHETIQUES

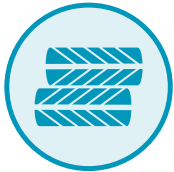

PNEUS

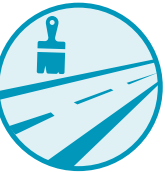

MARQUAGE ROUTIER

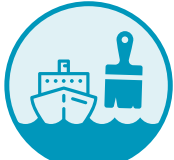

PEINTURES MARINES

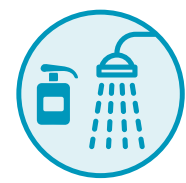

ARTICLES DE TOILETTE

\section{CONSOMMATION MONDIALE}

EN MILLIERS DE TONNES / AN

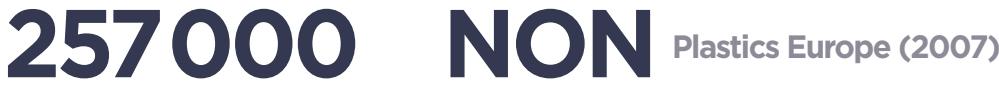

\section{NON FaAl'AaC (2013)}

\section{NON ERrma (2000)}

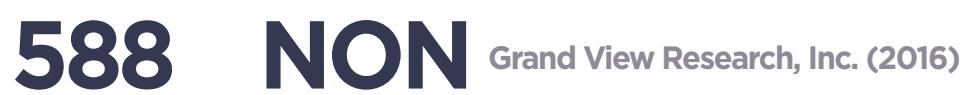

452 NON casings word (2002)

42 OUI Lesile, HA (2015) 


\section{Granulés de plastique : accidents pendant la fabrication, le transport et le recyclage}

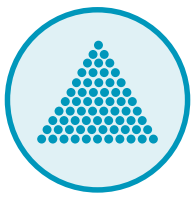

De nombreux plastiques débutent leur cycle de vie sous la forme de granulés, généralement de 2 à 5 mm de diamètre, ou de poudre. Les granulés sont transportés vers les transformateurs qui fabriqueront les objets en plastique. Tout au long de la chaîne de valeur du plastique, soit pendant la fabrication, la transformation, le transport et le recyclage, ces granulés peuvent être déversés accidentellement en plus ou moins grande quantité dans l'environnement (Essel et al., 2015). De nombreuses études de terrain rapportent ainsi la présence de granulés de plastique dans l'environnement. Ils sont aussi connus sous le nom de billes, paillettes et même larmes de sirène (Sundt et al., 2014).

Type de perte : involontaire

Étape du cycle de vie : production et transport de plastique primaire, recyclage de plastique

\section{Textiles synthétiques : usure pendant le lavage}

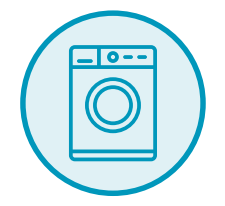

Le lavage des textiles synthétiques dans les blanchisseries industrielles et les foyers crée des microplastiques primaires par le frottement et la perte de fibres. Ces fibres passent dans les eaux usées (Browne et al., 2011) et peuvent terminer leur course dans l'océan (Magnuson et al., 2016).

Des quantités significatives de ces fibres textiles ont été observées lors d'études d'échantillonnage in situ à la fois en eau libre et dans des sédiments marins (Browne et al., 2011). Ce sont généralement des fibres de polyester, de polyéthylène, d'acrylique ou d'élasthanne (Essel et al., 2015).

Type de perte : involontaire

Étape du cycle de vie : entretien du produit

\section{Pneus : abrasion pendant le roulement}

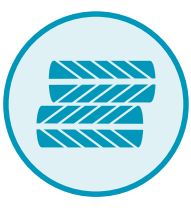

Les pneus s'érodent à mesure de leur utilisation, perdant des particules qui formaient leur enveloppe extérieure, qui se compose d'une matrice de polymères synthétiques dénommée caoutchouc styrènebutadiène (environ 60 \%) intégrée à un mélange de caoutchouc naturel et de nombreux autres additifs (Sundt et al., 2014). La poussière de pneus est ensuite dispersée par le vent ou emportée par les eaux de pluie. La présente étude ne tient compte que des pertes de caoutchouc synthétique, pas de caoutchouc naturel.

II n'existe pas d'informations fiables sur le transfert de microplastiques des pneus jusqu'aux océans. Des chercheurs norvégiens et suédois ont montré qu'une forte proportion des particules retrouvées dans la mer semblent provenir de pneus de voitures (Essel et al., 2015; Sundt et al., 2014).

Type de perte : involontaire

Étape du cycle de vie : utilisation du produit

\section{Marquage routier : exposition aux intempéries et abrasion par les véhicules}

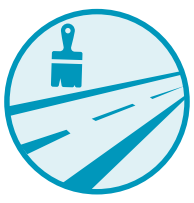

Le marquage au sol est appliqué pendant la construction et l'entretien des infrastructures routières. Il existe différents types de marquage (peinture, thermoplastique, bande de guidage en polymère préformé et peinture époxy), mais la majeure partie (45\%) est réalisée à la peinture (Grand View Research, Inc., 2016). Dans la plupart des pays européens, on utilise généralement des thermoplastiques (Lassen et al., 2015).

La perte de microplastiques résulte soit de l'exposition aux intempéries, soit de l'abrasion provoquée par le passage des véhicules. Comme la poussière de pneus, ces microplastiques sont dispersés par le vent ou emportés par les eaux de pluie avant d'atteindre les eaux de surface puis éventuellement les océans.

Type de perte : involontaire

Étape du cycle de vie : utilisation du produit 


\section{Peintures marines : exposition aux intempéries et accidents pendant l'application, l'entretien et l'élimination}

Les peintures marines sont appliquées sur toutes les pièces des bateaux pour les protéger, notamment la coque, la superstructure et les équipements du pont. II s'agit principalement de revêtements solides, de peinture anticorrosion ou de peinture antisalissures, qui peuvent se composer de plusieurs types de plastique, en général des peintures polyuréthane et époxy ainsi que des vinyles ou des laques (OECD, Series on emissions documents, 2009).

Les microplastiques primaires proviennent des activités de construction, d'entretien, de réparation ou d'utilisation des bateaux commerciaux ou de plaisance, en particulier des prétraitements de surface, de l'application de peintures et du nettoyage des équipements (OECD, Series on emissions documents, 2009).

Type de pertes : involontaire

Étape du cycle de vie : utilisation, entretien et élimination du produit

\section{Articles d'hygiène et de soin : versement dans l'eau pendant l'utilisation}

De nombreux produits d'hygiène et cosmétiques comptent parmi leurs ingrédients des microbilles de plastique qui servent à délivrer des ingrédients actifs, à exfolier ou à améliorer la viscosité du liquide. Certains de ces articles contiennent autant de plastique ajouté que le flacon dans lequel ils sont conditionnés (Leslie, 2015). Ce plastique peut représenter jusqu'à $10 \%$ du poids du produit et plusieurs milliers de microbilles par gramme de produit (Lassen et al., 2015).

L'utilisation normale de ces articles d'hygiène et de soin rejette directement des particules de plastique dans les eaux usées des foyers, hôtels, hôpitaux et infrastructures sportives, y compris les plages. Des microbilles provenant de tels produits ont été observées lors d'études de terrain dans différentes parties du monde (Driedger et al., 2015).

Type de pertes : volontaire

Étape du cycle de vie : utilisation du produit

\section{Poussières urbaines : expositions aux intempéries, abrasion et déversement}

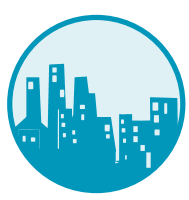

Le terme poussières urbaines désigne un groupe de neuf sources identifiées lors de récentes évaluations par pays et que l'on trouve principalement dans les environnements urbains (Essel et al., 2015; Lassen et al., 2015; Magnuson et al., 2016; Sundt et al., 2014). On trouve dans les poussières urbaines des particules issues de l'usure d'objets (semelles de chaussures, ustensiles de cuisine synthétiques), de la dégradation d'infrastructures (habitations, aménagements, pelouses artificielles, ports, y compris de plaisance, peintures des bâtiments) ainsi que de l'utilisation d'abrasifs ou de détergents. Ces sources sont regroupées, car leur contribution individuelle est faible. Cependant, elles représentent ensemble une quantité de rejets considérables à l'échelle d'un pays. À la différence d'autres sources qui ont fait l'objet de modélisations spécifiques par région, les poussières urbaines ont principalement été évaluées par extrapolation à partir d'études réalisées dans des pays scandinaves (Lassen et al., 2015; Magnuson et al., 2016; Sundt et al., 2014).

Type de pertes : principalement involontaires, mais en partie volontaires

Étape du cycle de vie : utilisation ou entretien du produit 


\subsection{Modélisation des activités, des pertes et des rejets}

Le présent rapport est basé sur la modélisation des sources et des rejets issus des activités économiques et domestiques. II s'appuie uniquement sur des données publiques disponibles et non sur des mesures de terrain.

La figure 2 décrit de manière générale l'approche adoptée pour calculer les pertes et les rejets dus à ces activités. Une présentation plus détaillée et des références figurent dans l'annexe méthodologique de ce rapport.

Pour chacune des activités, la quantité de plastique utilisée a d'abord été calculée pour les échelles régionale et mondiale. On a chiffré ensuite la quantité de microplastiques passant dans l'environnement (les pertes) en appliquant les taux de pertes propres à chaque activité et à chaque région. Enfin, la proportion de ces pertes terminant sa course dans l'océan (les rejets) a été calculée en appliquant les coefficients de transfert correspondants. Ces coefficients de transfert dépendent des quatre types de parcours distincts présentés cidessous et des différentes activités et régions:

- Océan : Iorsque les pertes ont lieu dans les océans (granulés de plastique, peintures marines), 100 \% de ces pertes sont des rejets.

- Eaux usées : Iorsque les pertes ont lieu le long des flux d'eaux usées (textiles synthétiques, articles de toilette et cosmétiques), le coefficient de transfert dépend de la couverture régionale et de l'efficacité du système de traitement des eaux usées.

- Ruissellement des eaux : Iorsque les pertes ont lieu sur les routes (granulés de plastique, pneus, marquage routier), une partie de ces pertes est dispersée par le vent alors que le reste est évacué par les pluies. Dans ce cas, le coefficient de transfert dépend du pourcentage des routes reliées à un drain séparé et de celles reliées aux systèmes d'assainissement.

- Vent : une fois perdus, les microplastiques peuvent être transportés jusqu'à l'océan par le vent.

Figure 2 :

\section{MODÉLISATION DES ACTIVITÉS, DES PERTES ET DES REJETS DE MICROPLASTIQUES PRIMAIRES}
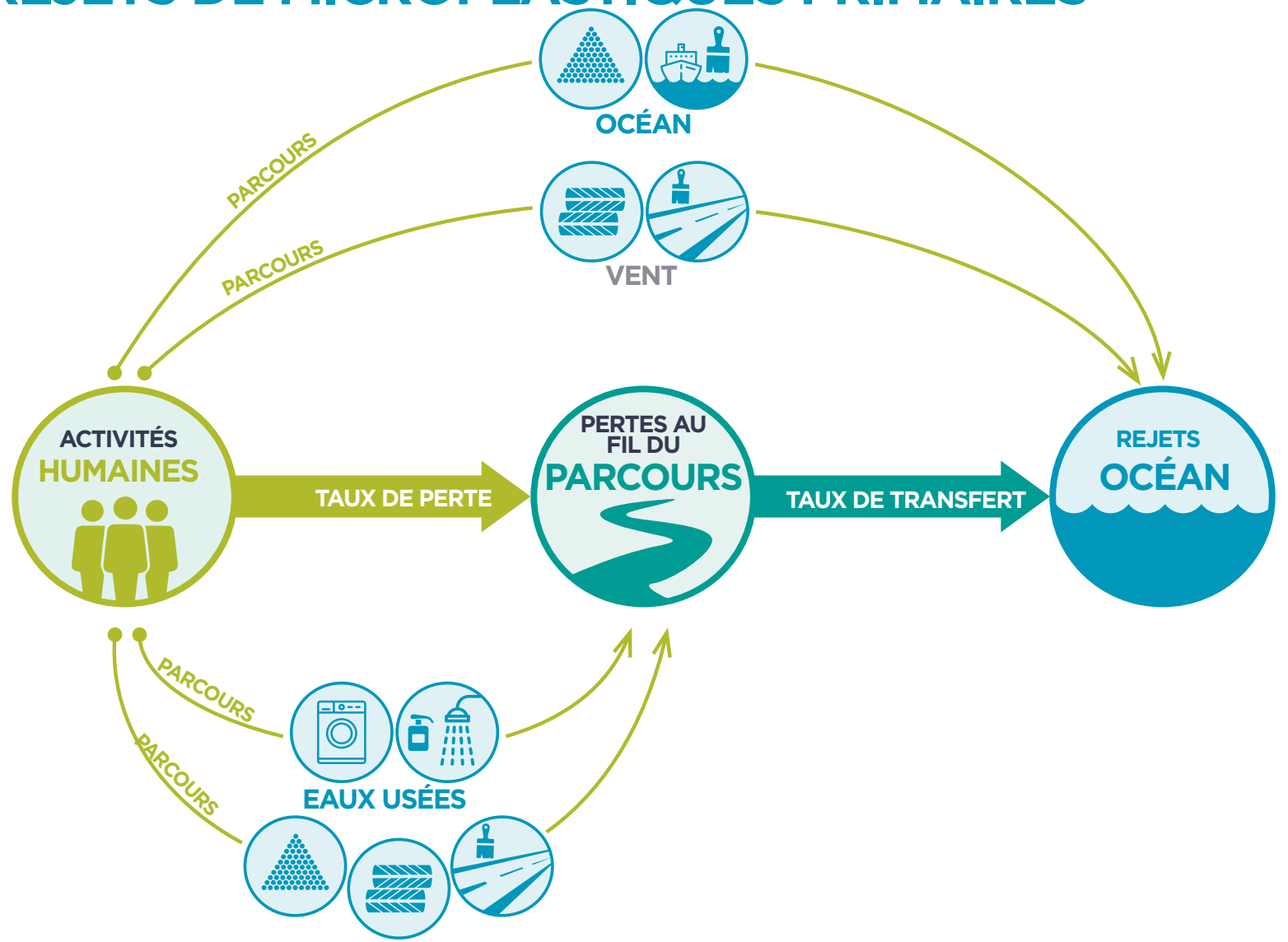

EAUX DE

RUISSELLEMENT 
Le chiffrage des activités, des pertes et des rejets a été réalisé de manière itérative. Dans un premier temps, nous avons estimé la pertinence globale de chacune des sources à l'échelle régionale. Pour toutes les sources à l'exception des poussières urbaines, l'estimation est fondée sur la multiplication des pertes génériques et des coefficients de transfert par la population et le PIB (produit intérieur brut) de la région.

Pour les poussières urbaines, une valeur globale a été évaluée à partir des pertes figurant dans les études quantitatives par pays (Lassen et al., 2015; Magnuson et al., 2016; Sundt et al., 2014). Cette valeur globale a ensuite été répartie par région à proportion des pertes calculées précédemment. Pour ce qui concerne la modélisation, différentes approches ont été testées et comparées afin de vérifier la fiabilité de notre modèle (voir l'annexe 1).

Dans un second temps, les calculs ont été affinés par l'application de données plus détaillées au niveau des pays, selon la disponibilité de ces données. Nous avons également testé un éventail plus large d'hypothèses afin de produire une limite haute et une limite basse des résultats, via deux approches complémentaires : une première fondée sur les activités annuelles et une seconde fondée sur le cycle de vie.

\subsection{Régions géographiques prises en compte}

Nous avons regroupé les pays en sept régions: Afrique et Moyen-Orient, Chine, Asie de l'Est et Océanie, Europe et Asie centrale, Inde et Asie du Sud, Amérique du Nord et Amérique du Sud, comme il est indiqué de manière plus détaillée en annexe 3.

\subsection{Présentation des résultats selon trois scénarios}

Le présent rapport expose les résultats selon trois scénarios : optimiste, neutre et pessimiste pour chacune des sept sources, à la fois aux niveaux régional et mondial.

Ces scénarios correspondent à l'ensemble le plus crédible de résultats après l'application d'un éventail d'hypothèses au cours de l'étude (voir l'annexe 1). Nous avons élaboré les scénarios optimistes et pessimistes en sélectionnant les résultats minimums et maximums après élimination des combinaisons d'hypothèses extrêmes et irréalistes. Le scénario neutre correspond à la valeur moyenne fondée sur la distribution des résultats restants. 


\subsection{Les microplastiques primaires, une part substantielle du plastique des océans}

Nos estimations des rejets de microplastiques primaires au regard de nos trois scénarios sont les suivantes:

Ces rejets constituent une fraction des pertes de microplastiques primaires estimées:

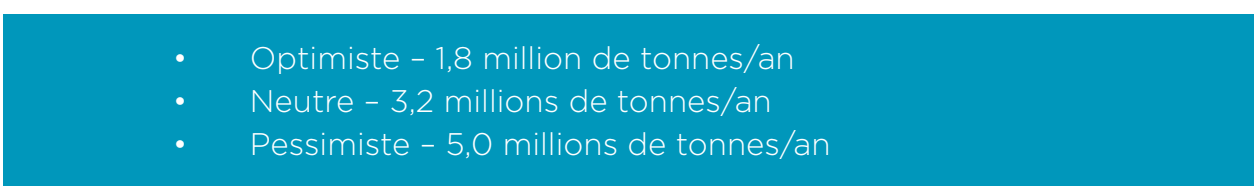

L'écart entre les pertes et les rejets dans le scénario neutre signifie que près de 48 \% des pertes de microplastiques primaires sont rejetées dans les océans. Le reste des microplastiques primaires est probablement piégé dans le sol ou les boues d'épuration. Leur sort et leurs effets à long terme demeurent inconnus et dépendent des circonstances et des pratiques ayant cours dans chaque région.

La comparaison entre ces rejets de microplastiques primaires et les rejets de microplastiques secondaires (c'est-à-dire ceux qui proviennent de déchets en plastique et de filets de pêche perdus) indique que la part de sources primaires pourrait être significative. La proportion de microplastiques primaires dépasse largement celle des filets de pêche perdus (0,6 million de tonnes par $a n^{4}$ ) (Circularocean, 2015; Macfadyen et al., 2009). Si I'on se fonde sur les valeurs calculées par Jambeck en 2015, les rejets issus de déchets en plastique pourraient représenter entre 4,8 et 12,7 millions de tonnes par an, soit en moyenne 8,0 millions de tonnes par an. Cela signifie, si l'on compare d'abord les valeurs neutres, puis les valeurs pessimistes, avec l'estimation des déchets la plus faible de ce dernier rapport, qu'entre 15 et 31 \% des microplastiques pourraient être d'origine primaire.

Nous avons décidé de centrer notre étude sur les pertes et les rejets de microplastiques d'origine pétrochimique uniquement. Les plastiques comme le caoutchouc naturel ne sont pas pris en compte. En élargissant notre point de vue et en supposant, à l'instar d'autres chercheurs européens (Essel et al., 2015; Lassen et al., 2015; Magnuson et al., 2016), que le caoutchouc naturel fait partie du problème, les rejets mondiaux de microplastiques augmenteraient de $45 \%, 33 \%$ et $26 \%$ pour chacun des scénarios. II en résulterait une valeur de 2 millions de tonnes par an dans le scénario neutre, avec une part estimée de microplastiques de $37 \%$.

Figure 3

\section{REJETS DE MICROPLASTIQUES DANS LES OCÉANS :}

\section{SOURCES PRIMAIRES ET SECONDAIRES (EN MILLIERS DE TONNES)}

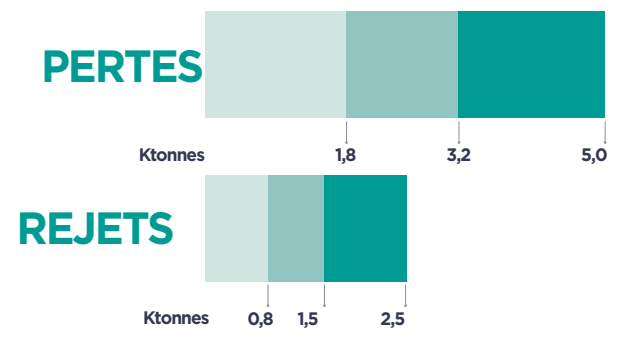

4 Cette valeur est la seule disponible, mais n'a pas été scientifiquement validée, elle doit donc être prise avec précaution. 
Nos résultats correspondent à ceux d'une recherche récente menée par Eunomia via une extrapolation très basique des rejets européens de microplastiques primaires (données issues d'un rapport précédent, breveté, d'Eunomia), élargis à l'échelle mondiale en utilisant le PIB par habitant exprimé en termes de parité de pouvoir d'achat. Eunomia estime que les rejets de microplastiques primaires représentent entre 0,5 et 1,41 million de tonnes par an, soit une valeur neutre de 0,95 million de tonnes par an (EUNOMIA, 2016b).

\subsection{L'usure de textiles et des pneus à l'origine de deux tiers des rejets}

Comme le montre la figure 4, relative au scénario neutre, près de deux tiers $(63,4 \%)$ des rejets sont issus d'abord du lavage de textiles synthétiques (34,8\%), puis de l'usure des pneus sur les routes (28,3\%). Cet ordre est le même dans le scénario pessimiste alors que dans le scénario optimiste, la part conjointe est similaire, mais la part due aux pneus est supérieure (38\%). Rappelons que ces résultats ne prennent en considération que le caoutchouc synthétique. En tenant compte du caoutchouc naturel, l'usure des pneus représenterait presque la moitié des rejets, soit 46,2 \% dans le scénario neutre.

La troisième part la plus importante (24,2 \%) est celle des poussières urbaines, qui a été calculée via une approche basique. II conviendrait d'affiner les études à cet égard pour mieux comprendre la contribution des poussières urbaines par région. Les articles d'hygiène et cosmétiques ne représentent que $2 \%$ des rejets mondiaux de microplastiques primaires dans les océans.

Figure 4

\section{REJETS DE MICROPLASTIQUES PRIMAIRES DANS LES OCÉANS}

\section{PAR SOURCE (EN \%).}
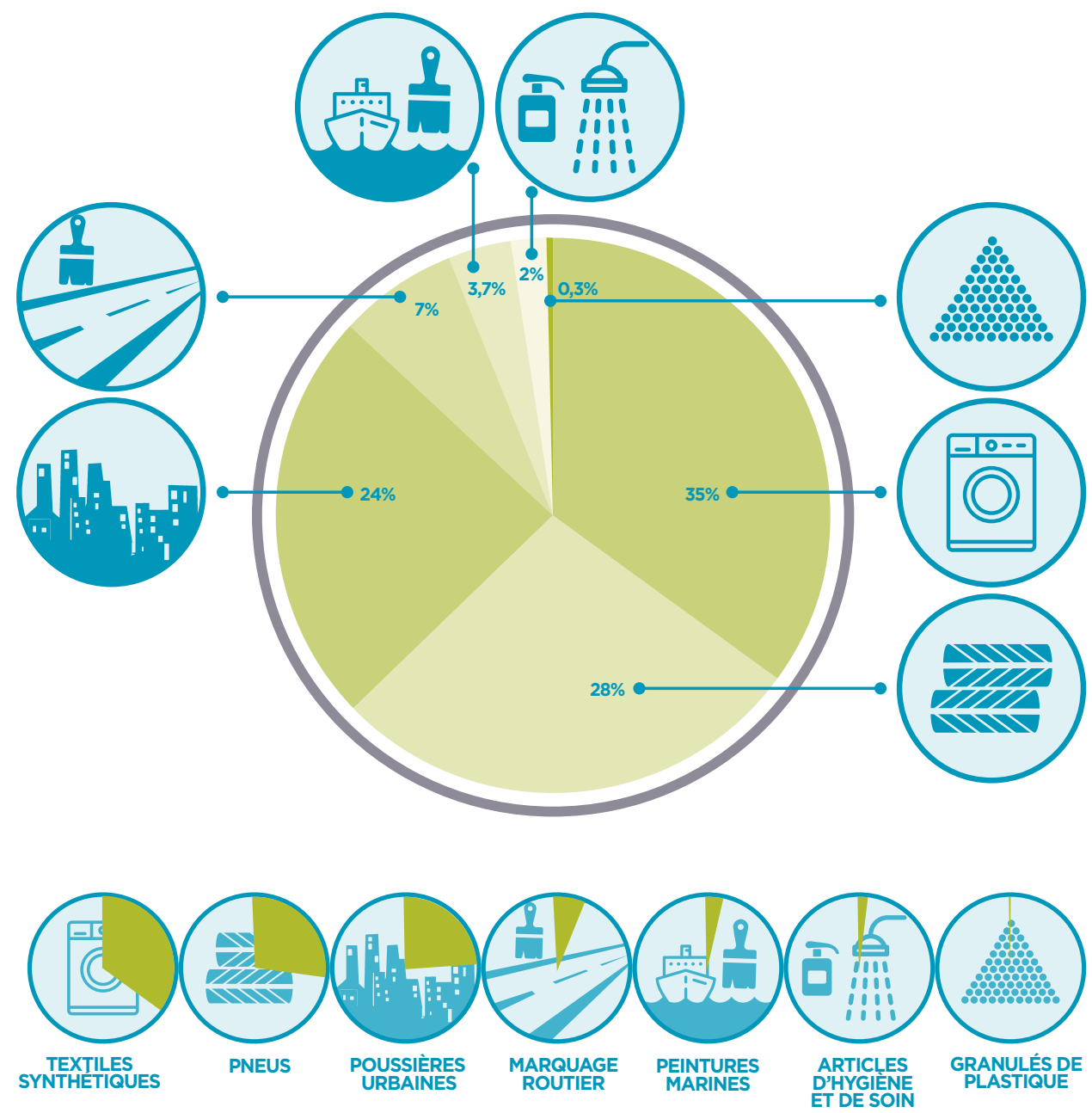


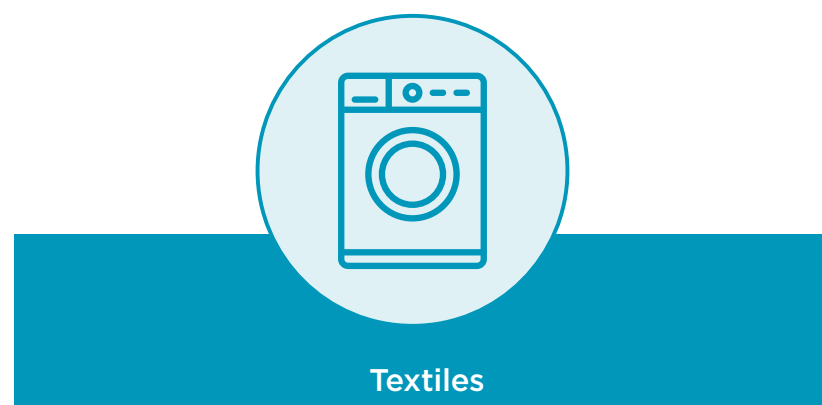

La consommation mondiale de fibres vestimentaires s'élève à 69,7 millions de tonnes par an, soit environ 11 kilos par habitant (2010), dont près des deux tiers sont des fibres synthétiques (60,1\%) (FAO/ICAC, 2013).

L'emploi de fibres textiles s'est progressivement accru au fil des deux dernières décennies (+79,3\% entre 1992 et 2010), une croissance presque exclusivement attribuable aux fibres synthétiques, dont la consommation est passée de 16 à 42 millions de tonnes sur cette période, soit une hausse de près de 300 \% (FAO/ICAC, 2013).

La majorité (62,7\%) des fibres synthétiques sont aujourd'hui utilisées dans les pays en développement, où les consommateurs achètent une plus grande proportion $(68,0 \%)$ de textiles synthétiques que dans les pays développés $(48,2 \%)$

La moyenne mondiale de la consommation annuelle par habitant, 6,1 kilos, cache d'importantes disparités entre les régions: 0,7 kg par personne en Afrique, $5,7 \mathrm{~kg}$ en Asie, et jusqu'à $17,8 \mathrm{~kg}$ en Amérique du Nord, soit un rapport de 1 à 25 (FAO/ ICAC, 2011).

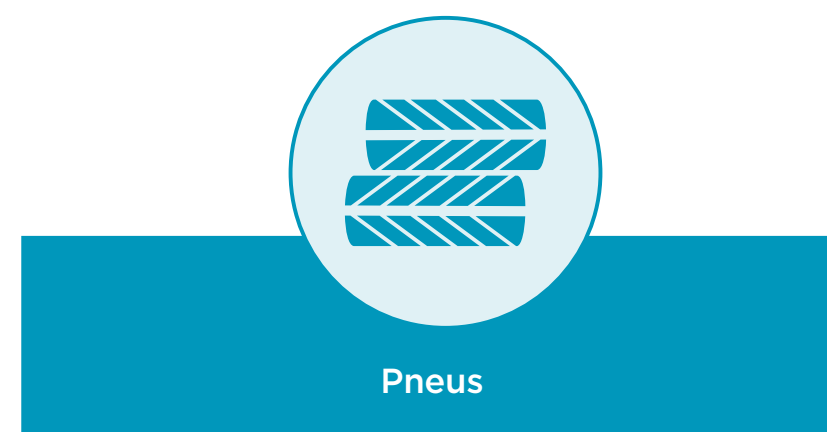

Le monde compte actuellement environ 1,41 milliard de véhicules, soit 0,3 par habitant en 2010 (ETRma, 2011). Plus des deux tiers (71,2\%) sont des voitures personnelles et des véhicules commerciaux légers, $25,6 \%$ sont des motos et $3,1 \%$ sont des véhicules commerciaux moyens et lourds. Ces chiffres ont augmenté progressivement entre 2000 et 2015 (78\%). La plupart des véhicules circulent en Asie (36\%), dont $13 \%$ en Chine. L'Europe compte $27 \%$ des véhicules en circulation et l'Amérique du Nord 21 \% (ETRma, 2011).

Tous ces véhicules sont pourvus de pneus en caoutchouc. $57 \%$ de l'ensemble du caoutchouc synthétique utilisé en 2010 ont servi à fabriquer des pneus. La consommation mondiale de caoutchouc synthétique a progressé de 27,3\% entre 2002 et 2010, notamment du fait de la Chine. Cette augmentation est inférieure à celle de la consommation mondiale de caoutchouc naturel sur la même période (+41,2%) (ETRma, 2009).

En 2010, il s'est vendu 13,9 millions de tonnes de caoutchouc destiné aux pneus, dont $46 \%$ de synthétique, soit 6,4 millions de tonnes (ETRma, 2011). La consommation moyenne mondiale de caoutchouc synthétique par habitant et par an, 0,93 kilos, cache d'importantes disparités entre les régions. 
4.3 Trois quarts des rejets domestiques dus à l'utilisation et l'entretien des produits

Figure 5

REJETS DE MICROPLASTIQUES DANS LES OCÉANS :

VENTILATION PAR TYPE D'ACTIVITÉS

ET PAR ÉTAPE DU CYCLE DE VIE

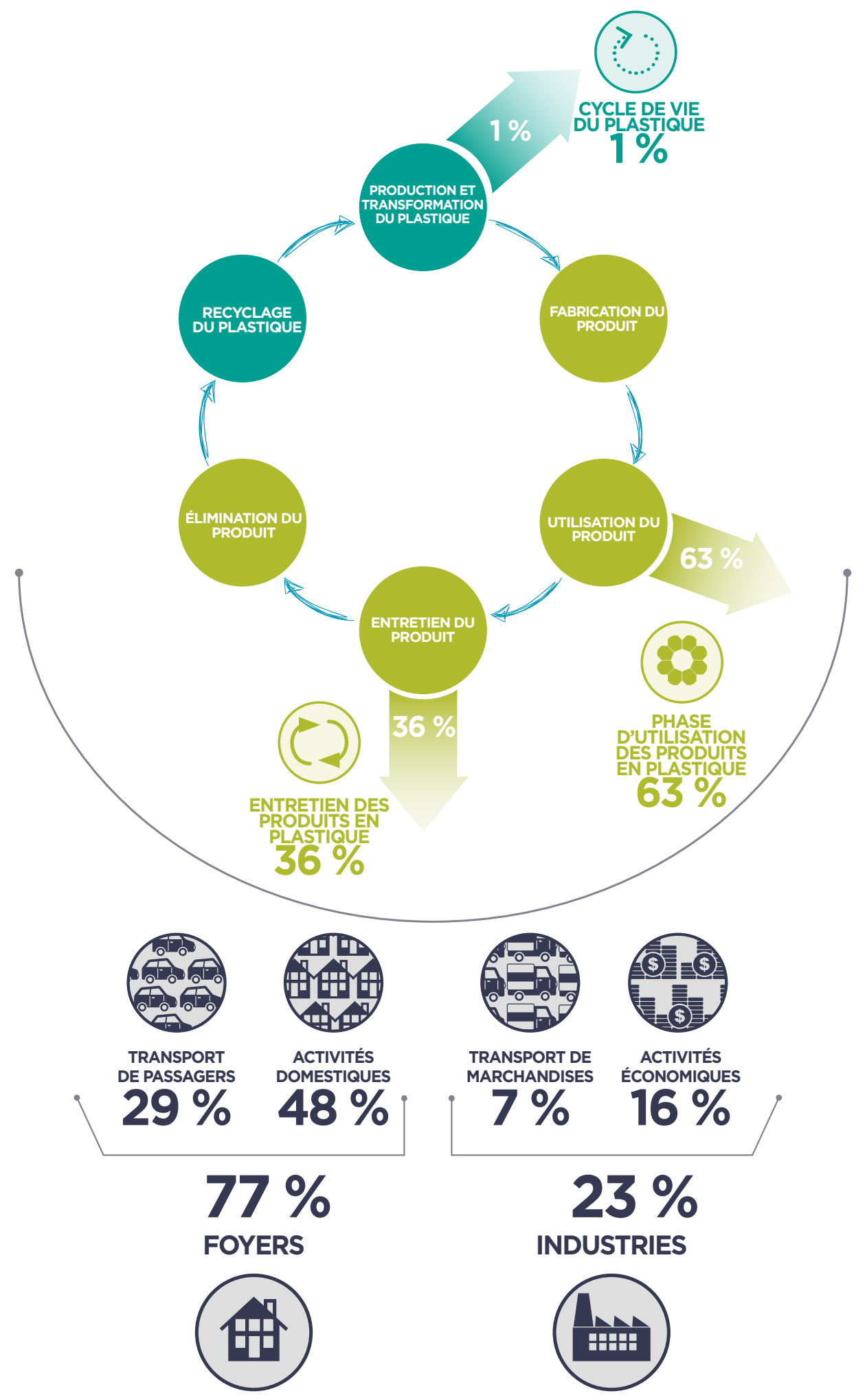


Comme le montre la figure 5, les foyers, par leurs activités, produisent près des trois quarts (77 \%) des rejets de microplastiques. Le reste procède des activités économiques. Les rejets provenant des foyers ont lieu majoritairement pendant la phase d'utilisation des objets (49\%) et le reste pendant leur entretien (28\%). Les activités de transport constituent le tiers (38 \%) des sources des rejets des foyers (transport de passagers) ${ }^{5}$ en raison de la contribution des pneus et du marquage routier, dus principalement aux voitures personnelles. En Europe, par exemple, $90 \%$ des distances parcourues le sont par des voitures personnelles (EU JRC/NL PBL, 2010). Le reste des sources (62\%) relève d'autres activités au sein des foyers, comme le lavage de textiles synthétiques, l'utilisation de produits d'hygiène et de soin et la peinture de bateaux de plaisance.

Les rejets procédant d'activités économiques sont donc bien plus faibles (23\%) que ceux générés par les foyers. La plupart d'entre eux se produisent pendant l'utilisation des objets tandis que la phase de fabrication, de transformation et de recyclage ne représente qu'entre 0,5\% et 1,5\%. La part des rejets attribuable au transport commercial est du même ordre que celle du transport individuel, bien que légèrement plus faible (30 \%). Le transport routier génère donc près d'un tiers (35,1\%) du total des rejets.

\subsection{Deux tiers des pertes dues aux eaux de ruissellement des routes}

L'écrasante majorité (98\%) des pertes de microplastiques primaires adviennent pendant des activités terrestres. Comme l'illustre la figure 6 , elles proviennent principalement (66 \%) des eaux de ruissellement des routes (pneus, marquage au sol et incidents impliquant des granulés de plastique sur terre), puis des systèmes de traitement des eaux usées (25\%) et enfin du vent (7\%). Les activités maritimes ne causent que 2 \% des pertes.

Pour ce qui concerne les rejets, la distribution est légèrement différente: au niveau mondial, près d'un tiers $(29,5 \%)$ de la population bénéficie directement d'un système de traitement des eaux usées. En raison des débordements, plus des deux tiers (71\%) des microplastiques contenus dans cette eau se retrouvent dans les océans. Les microplastiques contenus dans les eaux de ruissellement n'aboutissent dans l'océan que dans 32 \% des cas, notamment via des évacuations séparées. Enfin, toutes les pertes ayant lieu dans l'océan et celles disséminées par le vent deviennent des rejets ${ }^{6}$. Ainsi, $44 \%$ des rejets proviennent des eaux de ruissellement des routes, $37 \%$ des eaux usées, $15 \%$ ont été transportés par le vent et $4 \%$ sont des rejets directs dans l'océan.

Figure 6

\section{REJETS MONDIAUX DANS LES OCÉANS :}

\section{CONTRIBUTION DES DIFFÉRENTS PARCOURS AUX REJETS DE MICROPLASTIQUES}

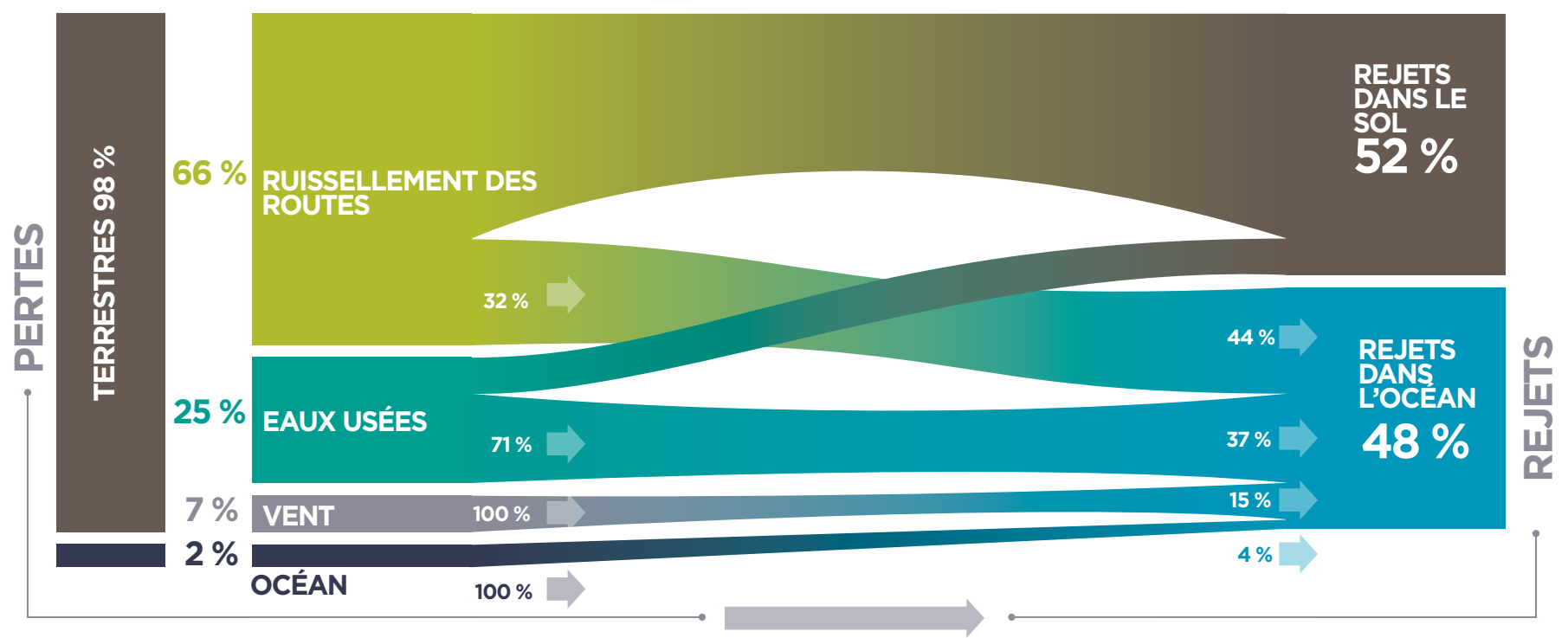

5 Pour l'usure des pneus et le marquage routier, la part attribuable aux foyers et celle attribuable aux activités commerciales ont été calculées sur la base des distances parcourues par les différents types de véhicules.

6 La proportion des particules transportées par le vent qui terminent leur course dans le sol est inconnue. 


\subsection{Une contribution significative de toutes les régions aux rejets}

La figure 7 décrit la répartition des rejets de microplastiques primaires, le PIB et la population par région et montre ainsi que la part de chacune des régions est significative: les plus gros contributeurs sont l'Inde et I'Asie du Sud (18,3\%), puis l'Amérique du Nord (17,2\%), suivis de près par l'Europe et l'Asie centrale (15,9\%), la Chine (15,8 \%) et l'Asie de l'Est et l'Océanie (15,0\%). Les rejets de l'Amérique du Sud (9,1 \%) et de la région Afrique et Moyen-Orient (8,7\%) sont plus faibles.

Ces rejets peuvent s'expliquer par une combinaison de trois facteurs, selon l'équation classique I=PAT (Impact $=$ Population $\times$ Richesse [Affluence] $\times$ Technologie disponible pour la production des biens) (Ehrlich and Holdren, 1971), c'est-à-dire en l'espèce le nombre d'habitants, le portefeuille d'activités générant des pertes (PIB par habitant) et la capacité des systèmes de traitement à retenir ces pertes.

En Inde et en Asie du Sud, en Chine et en Afrique et Moyen-Orient, les pertes par habitant sont inférieures à la moyenne mondiale (212 g/habitant/an). Les populations nombreuses dont une faible proportion bénéficie d'un système de traitement des eaux usées, par exemple en Inde et en Asie du Sud avec 6,2 \%, causent des rejets significatifs. L'Europe et l'Asie centrale ainsi que l'Amérique du Nord contribuent davantage aux rejets que la moyenne mondiale, mais pour des raisons exactement inverses : leur population est moins nombreuse, mais une plus grande proportion est reliée au système de traitement des eaux usées (71,4 \% en Amérique du Nord). L’Amérique du Sud, l'Asie de l'Est et l'Océanie se trouvent entre les deux.

Figure 7

\section{REJETS MONDIAUX DANS LES OCÉANS :}

\section{CONTRIBUTION DES DIFFÉRENTES RÉGIONS DU MONDE}

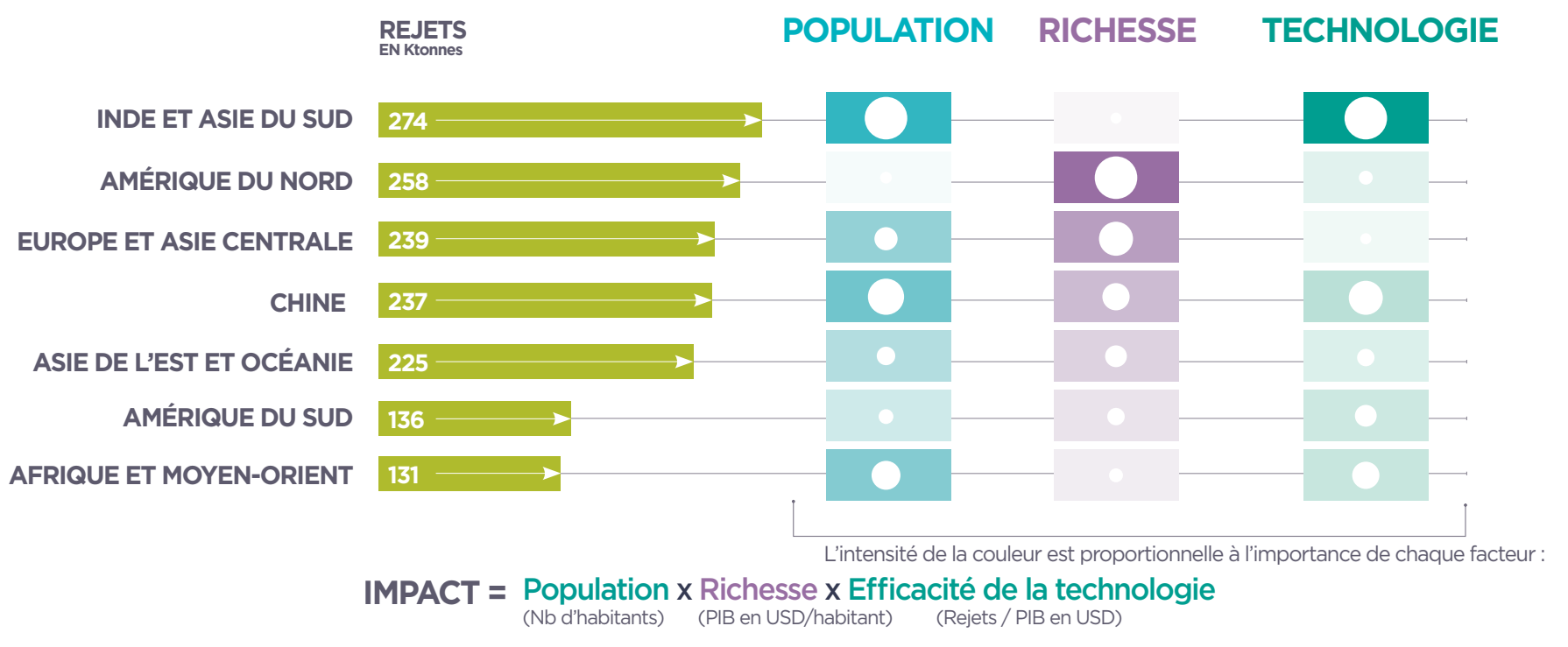




\subsection{Rejets moyens : un sac en plastique par personne et par semaine}

Les microplastiques primaires rejetés, soit 1,53 million de tonnes par an, équivalent à une moyenne de 212 grammes par an et par habitant? , soit 43 sacs d'épicerie légers 8 jetés dans l'océan par personne, c'est-àdire environ un par semaine.

Ce chiffre varie amplement d'une région à l'autre, comme l'illustre la figure 8, de 22 sacs en plastique par personne en Afrique et au Moyen-Orient à 150 en Amérique du Nord (soit un facteur d'un à sept).

Figure 8
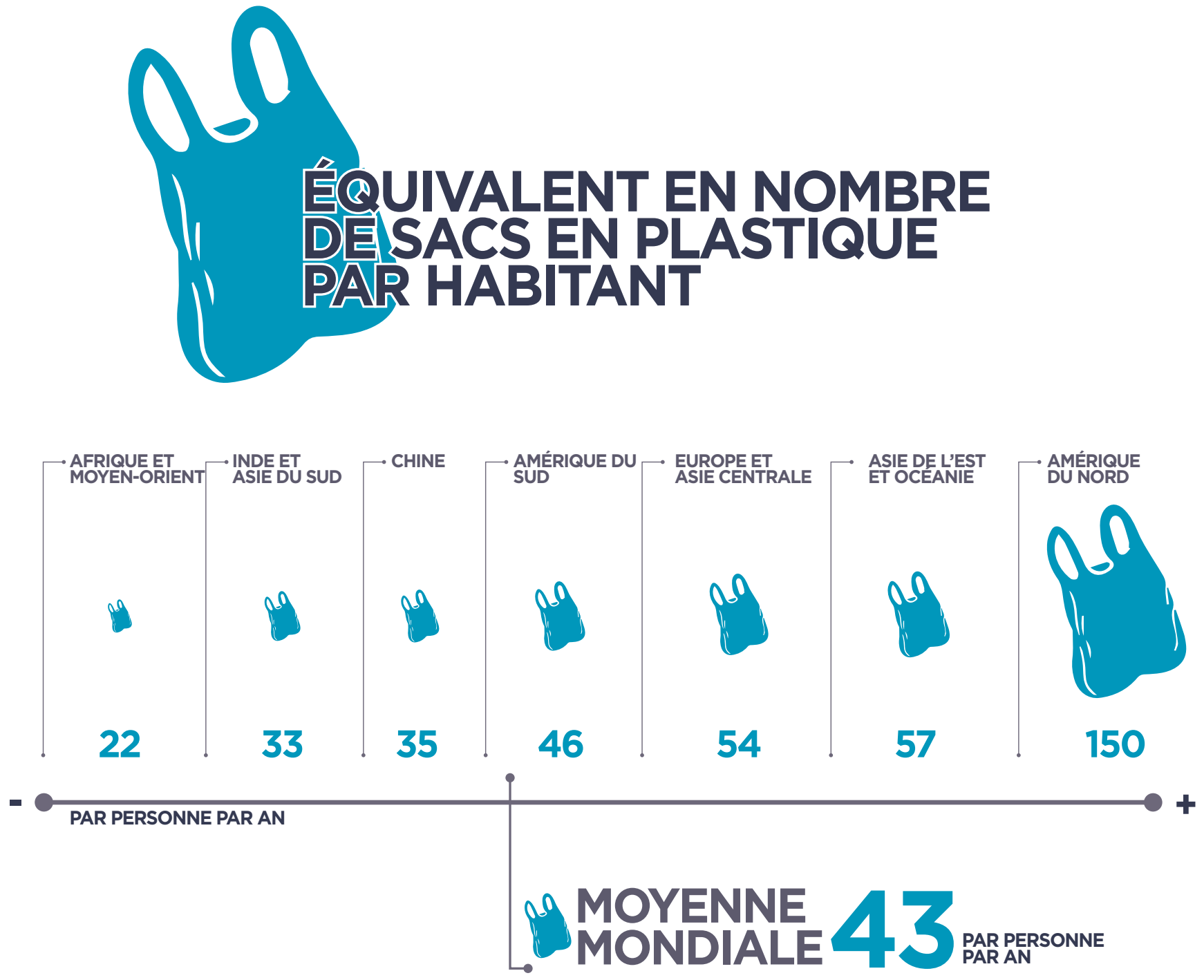

Population mondiale en 2012 


\subsection{Principales sources de rejets par région}

La figure 9 met en lumière l'importance relative de chacune des sources régionales au niveau mondial. Les textiles synthétiques constituent la principale source de microplastiques primaires en Asie, en Afrique et au Moyen-Orient. Dans ces régions, la part due aux vêtements synthétiques est supérieure à la moyenne mondiale, la proportion de la population bénéficiant d'un système de traitement des eaux usées est inférieure à la moyenne mondiale et la distance parcourue par des véhicules est inférieure à celle observée en Amérique (Nord et Sud) ainsi qu'en Europe et en Asie centrale.

Dans ces dernières régions, ce sont les pneus qui contribuent le plus aux rejets de microplastiques primaires : les distances parcourues par des véhicules sont bien plus élevées que dans le reste du monde, alors que les systèmes de traitement des eaux usées sont plus courants et retiennent donc une plus grande partie des microplastiques (par exemple ceux qui proviennent des textiles synthétiques) transportés par les eaux de ruissellement et le vent. Pour l'Europe, ces résultats correspondent aux constats effectués dans les pays scandinaves, où la plupart des pertes et des rejets sont attribuables aux pneus (Essel et al., 2015; Lassen et al., 2015; Magnuson et al., 2016; Sundt et al., 2014).

À l'échelle mondiale, les sources principales sont, par ordre d'importance (en pourcentage des rejets mondiaux, sans compter les poussières urbaines) : les textiles en Inde et Asie du Sud (15,9\%), les pneus en Amérique du Nord (11,5\%), les textiles en Chine (10,3\%) et les pneus en Europe et en Asie centrale (10,3\%).

Figure 9

\section{REJETS MONDIAUX DANS LES OCÉANS :}

PRINCIPALES SOURCES SELON LES RÉGIONS (TOTAL = $100 \%$ )

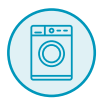

INDE ET ASIE DU SUD

AMÉRIQUE DU NORD

EUROPE ET ASIE CENTRALE

ASIE DE L'EST ET OCÉANIE

AMÉRIQUE DU SUD

AFRIQUE ET MOYEN-ORIENT

\section{4}

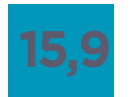

1,1

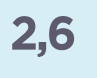

11,5
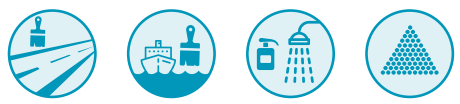

0,3

0,1

0,8

0

0,1

0,1

$1,9 \quad 1$

0,2

0,1

\section{4}

8,6

2,4

0,6

0,5

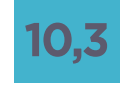

2,5

1,3

1,2

0,5

\section{6,3}

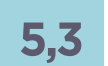

1,6

1,5

0,3

0

\section{2,9}

5,1

0,9

0

0,2

0

$\longrightarrow 3$

3,2

0,6

0,4

0,5

0
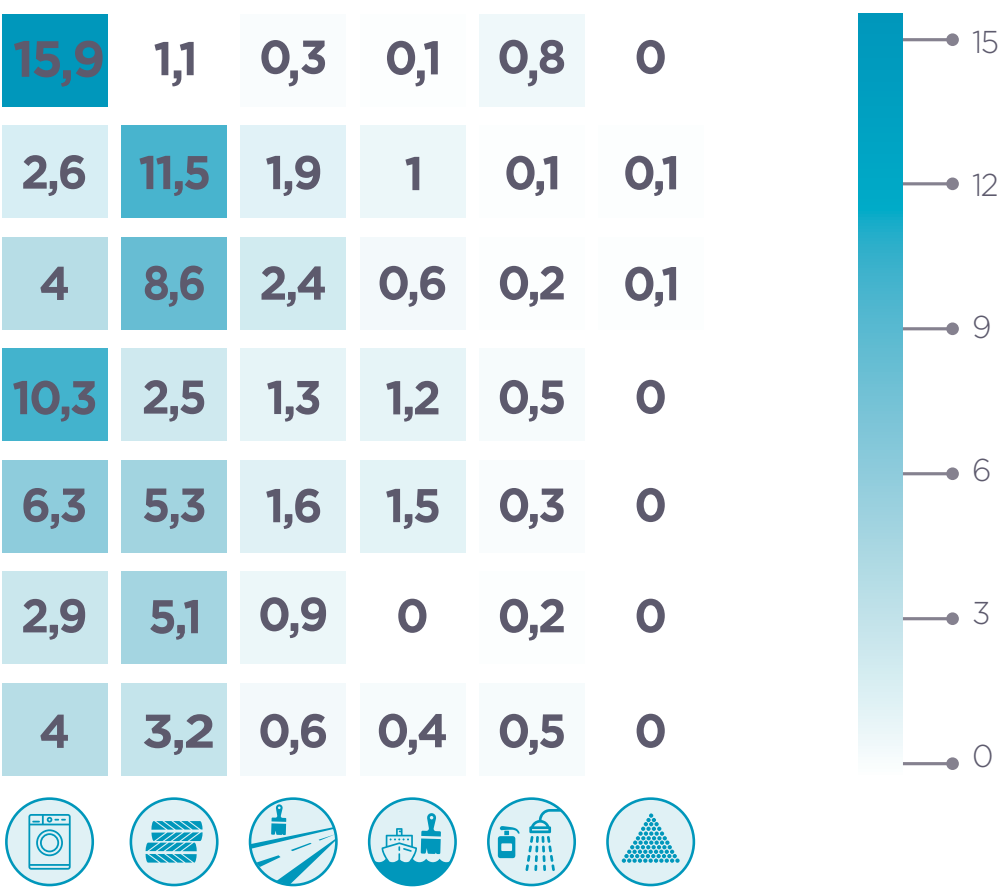


\subsection{Rejets de microplastiques élevés en Europe et en Amérique du Nord}

Outre la prépondérance des microplastiques primaires issus des pneus, les régions Amérique du Nord et Europe et Asie centrale ont pour particularité que leurs rejets de microplastiques primaires valent ou dépassent leurs rejets de microplastiques secondaires dus à la mauvaise gestion des déchets, ce qui n'est pas le cas dans les autres régions.

Figure 10

\section{REJETS MONDIAUX DANS LES OCÉANS :}

\section{COMPARAISON AVEC LES PLASTIQUES ISSUS DE DÉCHETS MAL GÉRÉS}

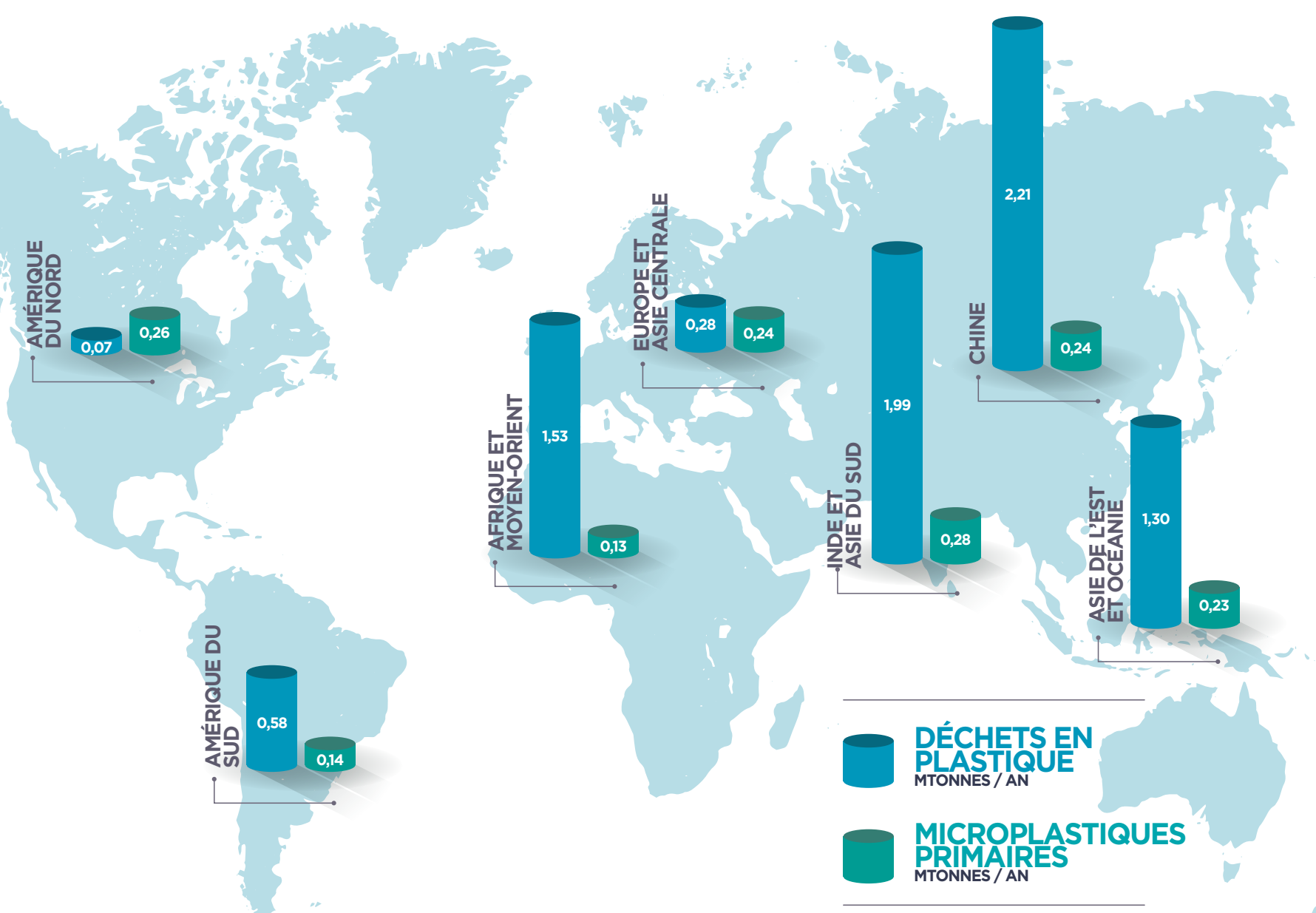




\subsection{Nécessité de varier les solutions pour stopper le flot de plastique}

Cette étude démontre clairement que les microplastiques primaires constituent une part considérable du plastique présent dans les océans. Par conséquent, les actions visant à juguler ce flot de plastique ne doivent pas seulement se concentrer sur une meilleure gestion des déchets dans certaines régions, mais aussi sur la réduction des pertes pendant le cycle de vie de certains produits. Les priorités pourront donc être différentes selon les régions.

Pour les endroits où les rejets de plastique se composent principalement de déchets mal gérés, il convient d'améliorer en priorité la gestion des déchets et de changer les habitudes. Mais au-delà d'une action classique en bout de chaîne, il serait sans doute opportun de réduire la production de plastique en optimisant, en réduisant et en réutilisant les emballages et d'accroitre les taux de recyclage, par exemple en valorisant les déchets en plastique.

Pour les régions où les rejets de microplastiques primaires égalent ou dépassent les rejets de microplastiques secondaires provenant des déchets, il convient d'adopter des solutions très différentes, notamment centrées sur la conception des produits et des infrastructures, les habitudes de consommation, les unes n'étant évidemment pas exclusives des autres:

- Dans le cas des pertes volontaires, comme celles causées par les articles d'hygiène et de soin, les solutions pourraient se trouver dans le retrait et le remplacement des microbilles en plastique. Plusieurs marques travaillent à leur substitution, ce qui permettra, espérons-le, de tarir en quelques années cette source de microplastiques ${ }^{9}$.

- Dans le cas des pertes qui surviennent pendant l'utilisation ou l'entretien du produit, il conviendrait d'élaborer des solutions qui impliquent tous les acteurs du cycle de vie du produit, des concepteurs aux ingénieurs chargés des systèmes de traitement des eaux usées. Nous prenons ci-dessous l'exemple des textiles et des pneus.

\section{Solutions potentielles de réduction des rejets issus des textiles synthétiques}

Transformation du plastique et fabrication du produit (chimiste/concepteur) : concevoir des fibres textiles qui ne se dispersent pas.

Fabrication du produit (concepteur) : prélaver les textiles pour éviter les pertes importantes du premier lavage.

Entretien des produits (concepteur de machines à laver): installer des filtres plus efficaces sur les machines.

Infrastructures d'assainissement : améliorer l'efficacité des traitements et filtrages.

Utilisation du produit (consommateur) : réduire la part de textiles synthétiques en changeant les habitudes de consommation. L'utilisation de fibres recyclées pourrait réduire les pertes de fibres en plus d'offrir un nouveau débouché à des déchets en plastique qui auraient pu devenir des rejets dans l'océan.

\section{Solutions potentielles de réduction des rejets issus des pneus}

Transformation du plastique et fabrication du produit (chimiste/concepteur) : concevoir des polymères de caoutchouc et des pneus de manière écologique pour réduire l'abrasion.

Utilisation du produit (concepteur des routes/de l'asphalte) : concevoir des revêtements routiers de manière à réduire l'abrasion.

Utilisation du produit (concepteur de l'infrastructure de traitement des eaux de ruissellement) : assurer une collecte des eaux et une séparation des plastiques adéquates.

Infrastructures d'assainissement : améliorer l'efficacité des traitements et filtrages.

9 Pour un exemple au Royaume-Uni, voir : http://www.bbc.com/news/uk-37263087 

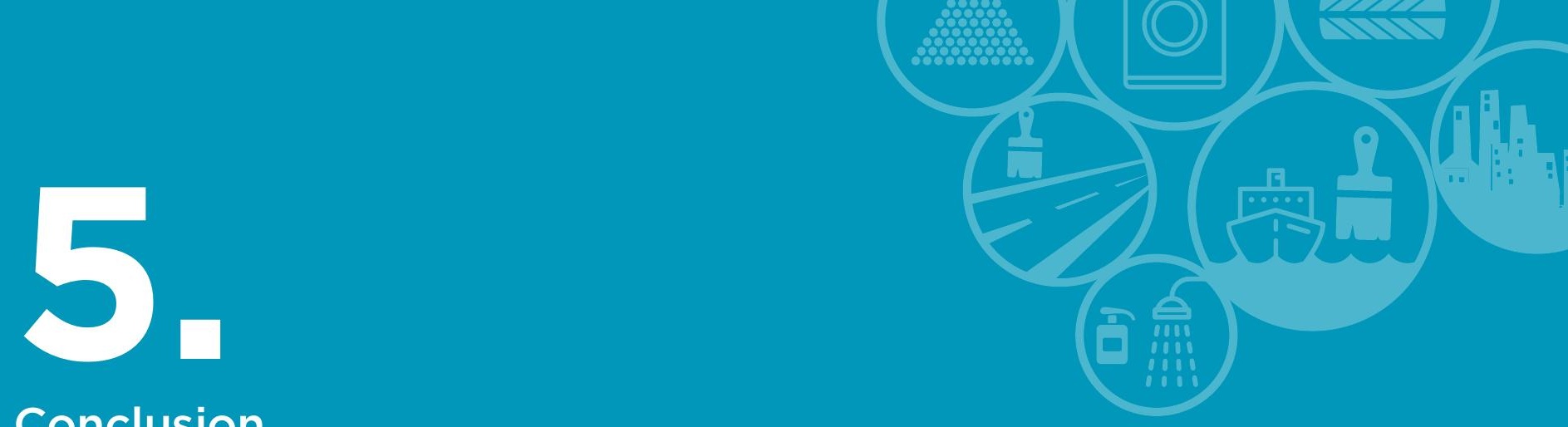

\section{Conclusion}

Cette étude est l'une des premières à démontrer que les microplastiques primaires contribuent largement à la présence de plastique dans les océans, avec des rejets mondiaux compris entre 0,8 et 2,5 millions de tonnes par an (valeur neutre: 1,5), soit, dans certaines régions, davantage que les microplastiques secondaires provenant des déchets mal gérés.
Si la réduction des déchets en plastique mal pris en charge reste une priorité à l'échelle mondiale, il est nécessaire de trouver également des solutions au problème des rejets de microplastiques primaires, notamment dans certaines régions et certains secteurs. Ces solutions exigeront l'adoption d'une approche systémique de la gestion du cycle de vie du produit, plutôt qu'une approche purement centrée sur la gestion des déchets. L'écoconception des objets et l'implication de tous les acteurs, du concepteur à l'urbaniste, du secteur public comme du secteur privé, seront au cœur de la stratégie qui permettra de tarir le flot de plastique.

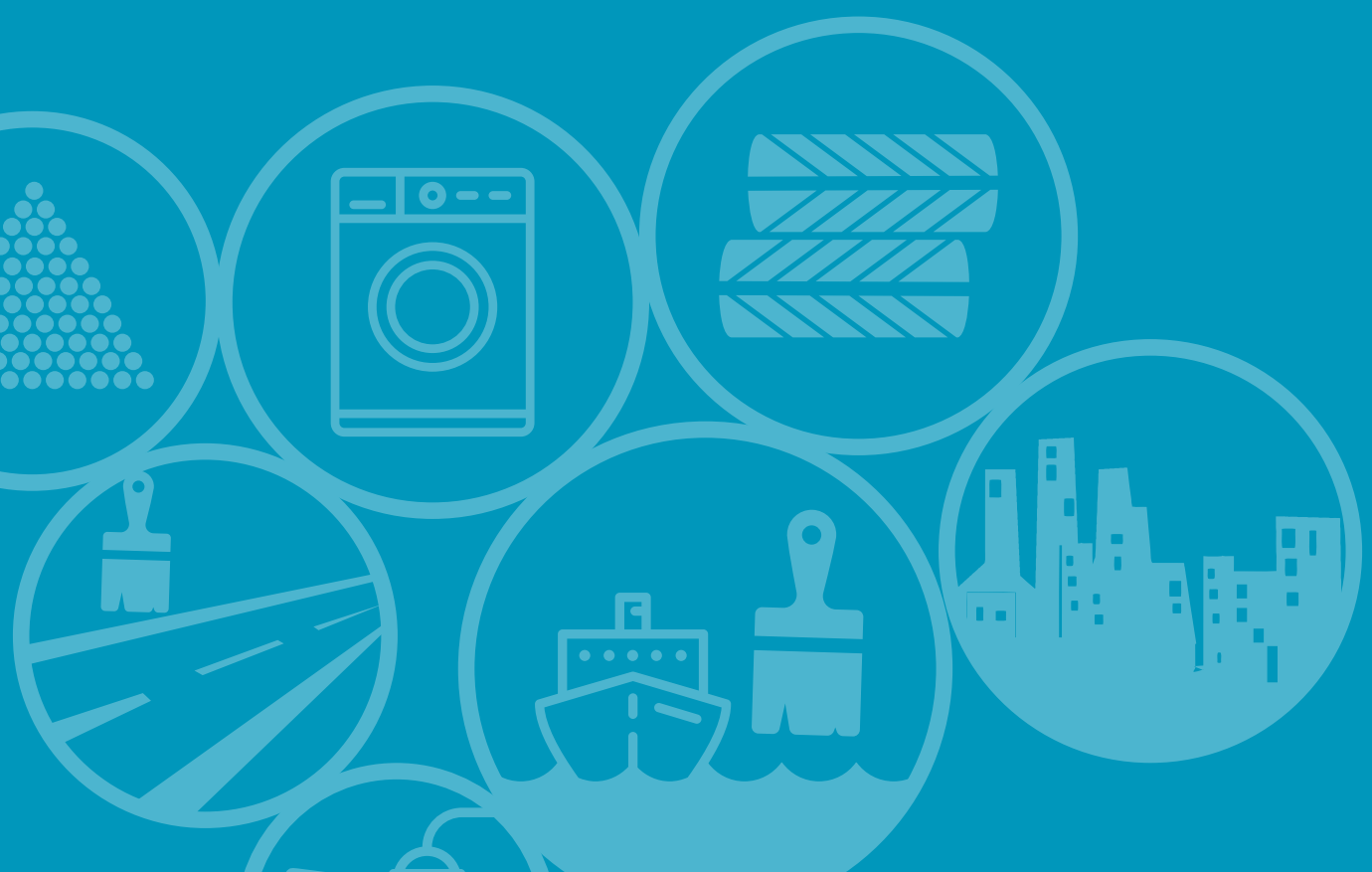




\subsection{Pour résumer}

Il est fort probable que :

- les pertes dans l'environnement de microplastiques primaires issus d'activités domestiques et commerciales représentent près de 3,2 Mtonnes/an;

- les origines principales de ces pertes soient l'abrasion des pneus sur les routes et l'usure des textiles synthétiques pendant le lavage (pertes diffuses pendant l'utilisation et l'entretien des produits);

- les rejets de microplastiques primaires représentent une part significative du plastique présent dans les océans;

- dans les pays à haut revenu disposant de systèmes de gestion des déchets convenables, les rejets de microplastiques primaires égalent ou dépassent les rejets provenant de déchets mal gérés. En revanche dans les pays à revenu plus faible, le plastique provenant de déchets mal gérés reste la source principale des rejets dans les océans.

II est plutôt probable que :

- les rejets dans l'océan de microplastiques issus d'activités commerciales et domestiques représentent près d'1,5 Mtonnes/an, soit $48 \%$ des pertes qui aboutissent dans l'océan;

- les pertes et rejets primaires augmentent dans les prochaines décennies, en raison de l'accroissement de la population et du niveau de vie dans les pays asiatiques et africains, à moins que des mesures ne soient prises pour réduire les taux de pertes (Impact = population $\times$ richesse $x$ technologie) (Ehrlich and Holdren, 1971). En dépit d'une meilleure gestion des déchets à travers le monde, la part de microplastiques primaires dans les rejets des pays en développement pourrait augmenter;

- les principaux parcours des microplastiques vers les rivières puis les océans soient les eaux usées et les eaux de ruissellement des routes;

- $\quad$ près de $52 \%$ des pertes de microplastiques soient piégées dans le sol lorsque les boues d'épuration sont utilisées comme fertilisants ou lorsque les particules sont emportées par les eaux de ruissellement des routes. Le sort et les effets à long terme de ces microplastiques dans le sol restent actuellement inconnus.

\subsection{Des mesures pour juguler le flot de plastique}

Si à l'échelle mondiale, mettre fin à la mauvaise gestion des déchets reste une priorité, pour de nombreuses régions et secteurs, il convient de trouver des solutions pour enrayer les rejets de microplastiques primaires.

La solution à la mauvaise gestion des déchets réside dans la mise en place des infrastructures et des bonnes pratiques nécessaires, ainsi que dans la sensibilisation des consommateurs. Les technologies existent et les difficultés sont plutôt d'ordre politique et économique.

L'arrêt des rejets de microplastiques primaires dans l'océan suppose des solutions différentes. Outre les articles d'hygiène et de soin, auxquels les microbilles sont ajoutées volontairement (et donc facilement retirables, comme le montre la tendance actuelle), la plupart des pertes sont involontaires et diffuses, ce qui les rend difficiles à enrayer par des solutions en bout de chaîne. Les pertes interviennent pendant l'utilisation et l'entretien des produits, généralement au sein des foyers, elles doivent donc être stoppées par l'adoption d'une perspective globale impliquant les fabricants et les consommateurs. L'abandon des microbilles dans les cosmétiques est une action salutaire, mais qui ne résoudra pas l'ensemble du problème (les cosmétiques ne représentent que $2 \%$ des rejets). II convient de ne pas négliger les textiles synthétiques et les pneus, qui contribuent bien plus que les cosmétiques aux rejets de microplastiques primaires dans les océans. 
Juguler le torrent de plastique supposera une autre conception et la mise en œuvre de solutions technologiques, politiques et comportementales, ciblant l'ensemble du cycle de vie du plastique et des produits pour réduire les pertes pendant leur fabrication, leur utilisation, leur entretien, leur élimination et enfin, éviter leur rejet dans l'océan.

Cette approche centrée sur l'écoconception requiert une gestion systémique du cycle de vie des produits et l'implication de tous les acteurs, qu'ils soient publics ou privés, de la conception des objets à l'aménagement du territoire comme l'ont montré d'autres études (UNEP SETAC, 2009).

Sur le principe qu'on ne peut améliorer que ce qu'on peut mesurer, il convient de définir des méthodes et des indicateurs pour fixer des objectifs et mesurer les progrès accomplis, y compris par leur intégration au sein de cadres et de politiques qui définissent déjà des cibles (comme les objectifs de développement durable), ainsi que d'outils plus opérationnels comme les analyses du cycle de vie. Ainsi, les entrepreneurs comme les concepteurs des produits garderont à l'esprit la question des microplastiques.

\subsection{Prochaines étapes}

L'étape à venir consistera à approfondir l'analyse présentée dans cette étude. Pour ce faire, il conviendra de recueillir des données supplémentaires et d'explorer plus avant les conséquences régionales et sectorielles. Nous avons également besoin de mieux comprendre les effets cumulatifs de ces rejets à long terme. En outre, il conviendra de valider notre modèle prédictif en le confrontant à des données empiriques provenant du terrain. Cela n'est actuellement pas faisable en raison de recherches lacunaires : il serait nécessaire d'élaborer de nouveaux dispositifs expérimentaux pour réaliser cette comparaison.

Bien que de nouvelles recherches soient en cours, la présente étude pourrait jouer un rôle essentiel dans l'ouverture de discussions sur les manières de mettre fin aux rejets de microplastiques sans se cantonner aux approches traditionnelles de la gestion des déchets. La présence croissante de microplastiques dans les océans requiert la tenue d'une nouvelle réflexion sur la réduction des rejets primaires comme secondaires tout au long de la chaîne logistique.

Plusieurs organisations, parmi lesquelles I'UICN ont entamé un dialogue avec les multiples parties prenantes en vue de tarir ce flot de plastique. Nous avons désormais besoin d'assurer que dans ce processus, la question des microplastiques primaires ne soit pas négligée au profit de celle des plastiques secondaires issus des déchets mal gérés. II sera pour cela nécessaire d'impliquer de nouveaux acteurs et d'adopter des approches novatrices de la gestion du cycle de vie des produits. 


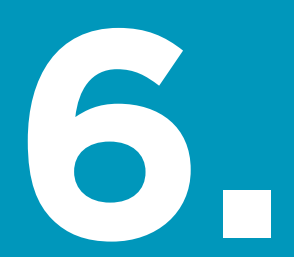

\section{Annexe 1 : Sources}

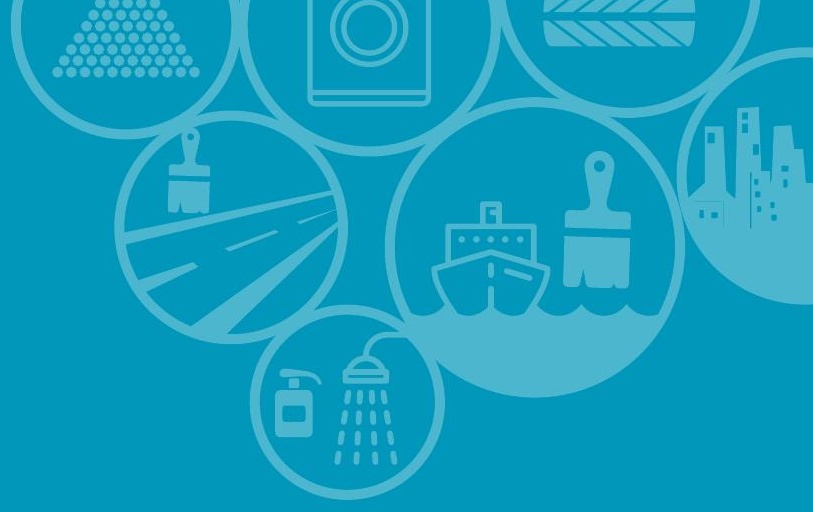

Les hypothèses retenues pour le calcul des scénarios optimiste, neutre et pessimiste sont présentées ciaprès par source, classées par ordre décroissant selon l'importance de leur contribution. Nous avons sélectionné ces scénarios, car ils constituent les éléments les plus crédibles de notre ensemble de résultats. Nous publierons séparément l'intégralité de nos hypothèses, de nos scénarios et de nos résultats.

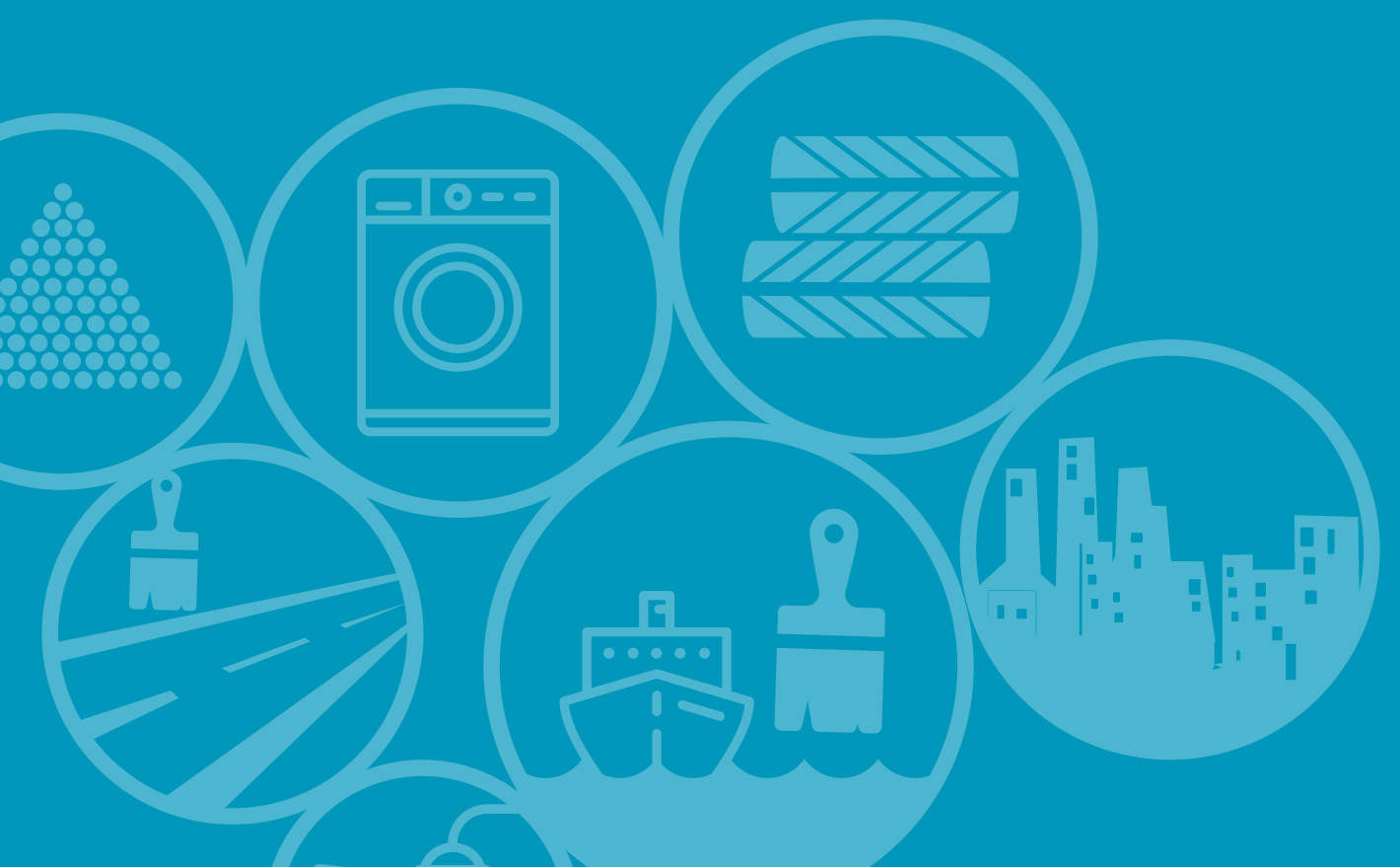




\subsection{Textiles synthétiques : usure pendant le lavage}

Les activités et les pertes sont calculées de deux manières : la première approche consiste à multiplier le nombre estimé de lavages par région par la quantité estimée de microplastiques perdus par lavage, telle que mesurée dans les eaux usées des machines à laver. La seconde approche consiste à multiplier les données relatives aux ventes annuelles mondiales et régionales de textiles synthétiques par les pertes globales estimées tout au long du cycle de vie d'un vêtement en textile synthétique.

\section{Approche 1}

Activités : nombre annuel de cycles de lavage par habitant (55) et charge moyenne par lavage (4 kg) tirés de l'étude de Pakula et Stamminger (2010). Population régionale (2007) tirée du PNUD (2015). Les proportions de textile synthétique par région sont calculées par nous sur la base de l'étude de la FAO/ICAC de 2011 (voir approche 2).

Pertes: estimations pessimiste/optimiste: $300 / 1500 \mathrm{mg}$ de microplastiques perdus par kilo de textile synthétique par lavage, selon l'étude de Lassen et al., de 2015. La valeur neutre est fixée à 900 mg/kg.

Rejets : voir le parcours des eaux usées en annexe 2.

\section{Approche 2}

Activités : consommation mondiale et régionale (2007) de textiles selon la FAO/ICAC, 2011. La correction des données et l'extrapolation aux sous-régions sur la base de la consommation par habitant et par l'ensemble de la population sont tirées du PNUD (UNDP, 2015).

Pertes : estimation pessimiste/optimiste: $0,74 / 5 \%$ de microplastiques perdus au cours du cycle de vie du produit (Essel et al., 2015; Lassen et al., 2015). La valeur neutre est fixée à $2 \%$.

Rejets : voir le parcours des eaux usées en annexe 2.

\section{Comparaison des deux approches}

Dans le scénario optimiste, les rejets estimés sur la base des faibles valeurs appliquées aux pertes dans la première approche correspondent à la valeur la plus faible appliquée dans la seconde approche ( $\pm 13 \%$ ). L'approche neutre est fondée sur une moyenne des scénarios neutres des première et seconde approches : les deux ne diffèrent que de $\pm 9 \%$ à l'échelle mondiale, mais concluent à une répartition différente selon les régions.

\subsection{Pneus : abrasion sur la route}

Les activités et les pertes sont calculées de deux manières. La première approche conjugue l'estimation de la distance parcourue par tous les véhicules d'une région et les émissions provenant des pneus estimées par kilomètre et par type de véhicule. La seconde approche conjugue les données relatives aux ventes mondiales et régionales de caoutchouc synthétique destiné à la fabrication des pneus et la moyenne des émissions produites par un pneu au cours de son cycle de vie.

\section{Approche 1}

Activités : distance annuelle parcourue, par pays (2011) pour le transport de marchandises et de passagers (OCDE, 2013). L'extrapolation de la distance aux autres pays a été réalisée sur la base d'un modèle de régression linéaire par la méthode classique des moindres carrés en utilisant les émissions de dioxyde de carbone par les transports (2005) (EU JRC/NL PBL, 2010). La part du transport de marchandises hors des pays de l'OCDE a été fixée à la moyenne pour l'OCDE (13\%). La part des poids lourds (par opposition aux camions légers) dans le transport de marchandises représente 0 \% dans le scénario optimiste, 50 \% dans le scénario neutre et $100 \%$ dans le scénario pessimiste. La part de caoutchouc synthétique prise en compte par pneu est celle qui avait été relevée en 2010, soit 46 \% (ETRma, 2011). La part des zones urbaines et des zones rurales est établie à 50 \% (scénario optimiste) ou fondée sur la part de la population vivant en zone urbaine (scénarios neutre et pessimiste) calculée par l'ONU-Habitat (Programme des Nations unies pour les établissements humains, 2016). 
Pertes : estimations optimiste/neutre/pessimiste: 0,033/0,051/0,178 gramme par pneu par kilomètre pour les voitures, les camions légers et les poids lourds (GRPE, 2013; Lassen et al., 2015; Sundt et al., 2014).

Rejets : voir le parcours des eaux de ruissellement en annexe 2.

\section{Approche 2}

Activités : consommation mondiale de caoutchouc synthétique destiné à la fabrication de pneus en 2010 (ETRma, 2011). La ventilation par région est fondée sur a) le nombre de véhicules par type (motos, voitures, véhicules commerciaux légers, moyens et poids lourds) par région en 2010 (ETRma, 2011), b) le nombre de roues par type de véhicule, soit respectivement 2, 4 ou 6, c) le poids moyen d'un pneu selon son type, soit respectivement 10, 16 et $50 \mathrm{~kg}$ (valeurs sélectionnées dans la littérature scientifique) et d) une proportion de caoutchouc égale à 25 \% (bande de roulement seule) par pneu (WRAP, 2006). La proportion de caoutchouc synthétique prise en compte par pneu est la même que dans la première approche.

Pertes : estimations optimiste/pessimiste : 10/25 \% de pertes de microplastiques au fil du cycle de vie (Essel et al., 2015; Magnuson et al., 2016 ; Sundt et al., 2014). La valeur neutre est fixée à 20 \%, soit l'équivalent de la perte de caoutchouc supposée au niveau mondial (quantité de caoutchouc synthétique calculée dans la première approche divisée par la quantité de caoutchouc synthétique vendue pour fabriquer des pneus).

Rejets : voir le parcours des eaux de ruissellement en annexe 2.

\section{Comparaison des approches des scénarios sélectionnés}

Les rejets estimés dans le scénario optimiste sur la base des faibles valeurs appliquées aux pertes calculées dans la première approche correspondent à la valeur la plus faible appliquée dans la seconde approche $( \pm 1,5 \%)$. Dans le scénario neutre, qui repose sur les valeurs neutres de pertes calculées dans la première approche, les rejets correspondent à la valeur centrale appliquée dans la seconde approche ( $\pm 9 \%)$. Dans le scénario pessimiste, l'estimation élevée de pertes de la première approche est inférieure de $37 \%$ à celle de la seconde approche.

\subsection{Poussières urbaines : déversements, exposition aux intempéries et abrasion}

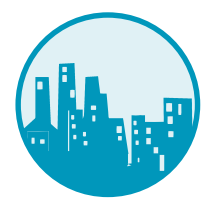

La modélisation concernant les poussières urbaines a été faite d'une manière différente et moins précise que pour les autres sources.

Poussières urbaines est la dénomination générique donnée à un groupe de neuf sources, identifiées lors d'évaluations récentes par pays, qui se trouvent généralement dans les environnements urbains (Essel et al., 2015; Lassen et al., 2015; Magnuson et al., 2016; Sundt et al., 2014). Ces sources ont été regroupées, car si leur contribution individuelle est faible, elles représentent ensemble une quantité considérable de pertes, mise en évidence dans les études par pays.

Activités : les poussières urbaines comprennent les pertes provenant de l'usure d'objets (semelles synthétiques de chaussures, ustensiles de cuisine synthétiques), de l'abrasion de l'infrastructure (poussières provenant des habitations, de la ville, pelouses artificielles, ports, y compris de plaisance, peintures des bâtiments), ainsi que les abrasifs de décapage et déversements volontaires (détergents). À la différence d'autres sources qui ont fait l'objet de modélisations précises par région, l'évaluation de la quantité de poussières urbaines est plutôt fondée sur une extrapolation basique à partir d'études réalisées dans des pays scandinaves. II conviendrait de procéder à une évaluation plus détaillée de cette source afin d’en préciser la pertinence.

Pertes: les pertes provenant d'autres sources ont été extrapolées (+ $29 \%$ à l'échelle mondiale) à partir des études suivantes: Lassen et al., 2015; Magnuson et al., 2016; Sundt et al., 2014), à proportion des pertes calculées pour les six sources principales.

Rejets : $50 \%$ des pertes sont acheminées vers l'océan par les eaux de ruissellement provenant du réseau routier et $50 \%$ par les eaux usées (notre hypothèse). 


\subsection{Marquage routier : exposition aux intempéries et abrasion par les véhicules}

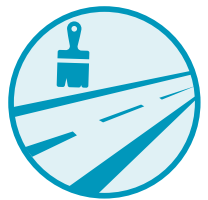

Les activités et les pertes ont été calculées en utilisant les données relatives à la consommation annuelle et régionale des produits de marquage au sol, la teneur moyenne en plastique de chaque produit de marquage (peinture, thermoplastique, bande de guidage en polymère préformé, époxy) et les pertes relevées dans les études existantes.

Description : la consommation mondiale de produits de marquage au sol s'élève à environ 1200 milliers de tonnes (2014), une quantité qui dépend du développement de l'infrastructure routière, de son entretien et de questions de sécurité. Le marché le plus important est l'Europe (31,9\%), suivie par les États-Unis. Différents types de marquage sont appliqués (peinture, thermoplastique, bande de guidage en polymère préformé), mais il s'agit majoritairement de peinture (44,7\%) (Grand View Research, Inc., 2016).

Activités: la consommation de produits de marquage dans le monde, aux États-Unis et en Europe (2014) provient de Grand View Research, Inc., 2016. L'évaluation de la consommation des autres régions, soit 43 \% des produits de marquage est basée sur le PIB (Banque mondiale, 2016). La part que représente la peinture dans le monde provient de Grand View Research, Inc., 2016, de même que la part des autres produits de marquage aux États-Unis. La teneur en plastique de chacun de ces produits est la suivante : peinture : 10,5/40 \% pour, respectivement, les scénarios optimistes et neutres ensemble et le scénario pessimiste (Lassen et al., 2015; Sundt et al., 2014), 25 \% pour les thermoplastiques (Lassen et al., 2015; Sundt et al., 2014) et 100 \% pour les autres produits (bande de guidage). Ces chiffres reprennent les mesures réalisées aux États-Unis et la part moyenne mondiale de la peinture. La répartition entre les zones urbaines et rurales est fondée sur l'estimation de la population urbaine par le Programme des Nations unies pour les établissements humains (2016).

Pertes: estimations optimiste/neutre/pessimiste: 23/43/100\%, tirées des estimations de Grand View Research (2016) des pertes de microplastiques (Sundt et al., 2014) (Lassen et al., 2015).

Rejets : voir le parcours des eaux de ruissellement en annexe 2.

\subsection{Peintures marines : exposition aux intempéries, application et entretien}

Les activités et les pertes sont calculées sur la base de la quantité de peinture marine appliquée chaque année dans le monde et par région, de la teneur en plastique de la peinture et des pertes estimées pendant la durée du cycle de vie des bateaux selon les études existantes sur ce sujet.

Activités: les applications mondiales et régionales de peinture marine (2012), ainsi que la part mondiale pour chaque type de bateau (porte-conteneurs, plaisance, cargos) et chaque type d'application (construction, entretien, réparation, révision) sont tirées de l'étude de Wright de 2009, ainsi que la répartition régionale, qui a été complétée par le PIB pour l'Asie (Banque mondiale, 2016) et par le nombre de bateaux de plaisance aux États-Unis et en Union européenne ${ }^{10}$ (Laaksonen, 2012). La teneur en plastique de la peinture est évaluée à 50 \% (Lassen et al., 2015).

Pertes : estimations optimiste/neutre/pessimiste du taux de perte pour les cargos : 3/6/9 \% (OECD Series on emissions documents, 2009) pour la valeur neutre. Estimations optimiste/neutre/pessimiste du taux de pertes pour les bateaux de plaisance en Amérique du Nord et en Europe : 10/30/50 \%. Estimations optimiste/neutre et pessimiste du taux de pertes pour les bateaux de plaisance dans les pays en développement: 50/90 \% (Sundt et al., 2014).

Rejets : voir le parcours des océans en annexe 2. 


\subsection{Articles d'hygiène et de soin : pertes pendant l'utilisation}

Les activités et les pertes sont calculées sur la base du nombre d'habitants par région et des pertes par habitant annuelles en Europe relevées dans les études existantes.

Activités : nombre d'habitants par région (UNDP, 2015).

Pertes : estimations optimiste/neutre/pessimiste: 1/6/13 g par habitant par an (Gouin et al., 2015; Leslie, 2015; Essel et al., 2015).

Rejets : voir le parcours des eaux usées en annexe 2.

\subsection{Granulés de plastique : fabrication, transport et recyclage}

Les activités et les pertes sont calculées sur la base des quantités de plastique primaire produites annuellement dans le monde et par région, qui contribuent aux pertes au cours de leur fabrication, de leur transport sur mer ou sur terre et de leur fin de vie en tant que plastique et produits en plastique, telles qu'exposés dans les études existantes. Les rejets sont ventilés par région sur la base des produits en plastique, c'est-à-dire du point de vue du consommateur (empreinte plastique).

Activités: la production mondiale et régionale de plastiques primaires (2007) provient de PlasticsEurope, 2009. L'attribution des plastiques primaires aux sous-régions et aux pays est fondée sur le PIB. Le calcul de l'empreinte plastique par région (point de vue du consommateur) a été réalisé via un modèle d'entrées-sorties plurirégional global basé sur Exiobase 2.0 (Tukker et al., 2014).

Pertes : les pertes sont calculées à quatre étapes : production de plastiques primaires, fabrication de plastiques, transport sur terre (pour les utilisations de produits en plastique) et sur mer (pour les échanges interrégionaux de produits en plastique) ainsi que l'élimination et le recyclage du plastique. Estimations optimiste/neutre/ pessimiste : 0,000 03/0,0001/0,001\% de pertes de microplastiques par étape (Cole et Sherrington, 2016).

Rejets : transport sur terre de plastiques primaires et de plastiques à usage domestique : voir le parcours des eaux usées en annexe 2. Transport sur mer de plastiques primaires et de plastiques importés : voir le parcours des océans en annexe 2. 


\subsection{Parcours des eaux de ruissellement des infrastructures routières}

Où : pertes et rejets terrestres

Sources : pneus, marquage routier, granulés de plastique sur terre

Lorsque les pertes ont lieu sur les routes, une partie d'entre elles sont dispersées par le vent (voir le parcours des vents). L'autre partie est emportée par les eaux de ruissellement. On considère que dans les zones rurales, peu de routes sont reliées au système des égouts : on estime qu'en moyenne, 3,5 \% des pertes mondiales finissent leur course dans les océans (Lassen et al., 2015; Ten Broeke et al., 2008). Dans les zones urbaines, deux cas sont possibles : soit les eaux de ruissellement sont évacuées par un drain séparé, soit elles sont évacuées dans le système d'égouts. Dans le premier cas, on estime que $80 \%$ des pertes se retrouvent in fine dans les océans (Lassen et al., 2015). Dans le second cas, les rejets dans l'océan dépendent d'une hypothèse supplémentaire, à savoir la part des routes reliées au système des égouts. L'hypothèse pour le scénario optimiste est que 50 \% des routes sont reliées au système d'assainissement. L'hypothèse pour les scénarios neutre et pessimiste est fondée sur le rapport entre la part de la population dont le foyer est relié au système de traitement des eaux usées et la proportion de routes équipées d'un système de collecte des eaux de ruissellement. Le parcours des eaux usées est analysé ci-après.

\subsection{Parcours des eaux usées}

Où : pertes et rejets sur terre

Sources : textiles synthétiques, articles d'hygiène et de soin, granulés de plastique sur terre

Lorsque les pertes rejoignent les eaux usées, le taux de rejet dépend de la couverture régionale et de l'efficacité du système de traitement des eaux usées. Notre hypothèse est que le pourcentage d'eau traitée est proportionnel à la part de la population bénéficiant d'un système de traitement des eaux usées. Cette part par région est fondée sur un ensemble de données calculées par nous sur la base des informations de UNSTATS (Division statistique du secrétariat de l'ONU, 2011) et, pour les pays et les régions manquants, des données tirées de la littérature scientifique. L'efficacité du traitement des eaux usées à l'échelle mondiale a été évaluée à $85 \%$. Ce chiffre a servi à estimer la part de microplastiques non retenue par les systèmes de traitement des eaux usées (3-6\%), soit qu'il s'agisse de fibres trop petites, selon Lassen et al. (2015), soit en raison de débordements (10 \%) en Europe selon Phillips et al. (2012). Les débordements sont probablement beaucoup plus importants dans les pays en développement, mais les chiffres manquent.

\subsection{Parcours des vents}

Où : pertes et rejets sur terre

Sources : pneus et marquage routier

Lorsque les pertes ont lieu sur les routes, une partie d'entre elles est dispersée par le vent. Cette part est estimée à 10 \% sur la base de l'étude de Wang et al. (2015). 100 \% de ces pertes deviennent des rejets.

\subsection{Parcours des océans}

Où : pertes et rejets dans la mer

Sources : granulés de plastique, peintures marines

Lorsque des pertes se produisent dans les océans, $100 \%$ d'entre elles deviennent des rejets. 


\section{Bibliographie}

La Banque mondiale(2016). GDP (current US\$): World Bank national accounts data, and OECD National Accounts data files.

Browne, M.A., Crump, P., Niven, S.J., Teuten, E., Tonkin, A., Galloway, T., and Thompson, R. (2011). Accumulation of Microplastic on Shorelines Woldwide: Sources and Sinks. Environ. Sci. Technol. 45, 9175-9179.

https://doi.org/10.1021/es201811s

Circularocean (2015). Circularocean website http://www. circularocean.eu/.

Cole, G., and Sherrington, C. (2016). Study to Quantify Pellet Emissions in the UK, Eunomia, March 2016.

Driedger, A.G.J., Dürr, H.H., Mitchell, K., and Van Cappellen, P. (2015). Plastic debris in the Laurentian Great Lakes: A review. J. Gt. Lakes Res. 41, 9-19.

https://doi.org/10.1016/j.jglr.2014.12.020

Eerkes-Medrano, D., Thompson, R.C., and Aldridge, D.C. (2015). Microplastics in freshwater systems: A review of the emerging threats, identification of knowledge gaps and prioritisation of research needs. Water Res. 75, 63-82.

https://doi.org/10.1016/j.watres.2015.02.012

Ehrlich, P.R., and Holdren, J.P. (1971). Impact of Population Growth. Science 171, 1212-1217.

https://doi.org/10.1126/science.171.3977.1212

Eriksen, M., Lebreton, L.C.M., Carson, H.S., Thiel, M., Moore, C.J., Borerro, J.C., Galgani, F., Ryan, P.G., and Reisser, J. (2014). Plastic Pollution in the World's Oceans: More than 5 Trillion Plastic Pieces Weighing over 250,000 Tons Afloat at Sea. PloS One 9, e111913.

https://doi.org/10.1371/journal.pone.0111913

Essel, R., Engel, L., Carus, M., and Ahrens, R.H. (2015). Sources of microplastics relevant to marine protection in Germany, Publication as pdf: www.umweltbundesamt.de/ publikationen/sources-of-microplastics-relevant-to-marine, $45 \mathrm{pp}$.

ETRma (2009). European Tyre \& Rubber Industry. Statistics. Edition 2009. no1.

ETRma (2011). European Tyre \& Rubber Industry. Statistics. Edition 2011. no3.

EU JRC/NL PBL (2010). Emission Database for Global Atmospheric Research (EDGAR), release version 4.1.

EUNOMIA (2016a). Study to support the development of measures to combat a range of marine litter source (European Commission DG Env).

EUNOMIA (2016b). Plastics in the Marine Environment.

FAO/ICAC (2011). Summary of the World Apparel Fiber Consumption Survey - Edition 2011.
FAO/ICAC (2013). Summary of the World Apparel Fiber Consumption Survey - Update 2013.

GESAMP (2015). Sources, fate and effects of microplastics in the marine environnement: a global assessment (GESAMP).

Gouin, T., Avalos, J., Brunning, I., Brzuska, K., de Graaf, J., Kaumanns, T., Koning, T., Meyberg, M., Rettinger, K., Schlatter, H., et al. (2015). Use of Micro-Plastic Beads in Cosmetic Products in Europe and Their Estimated Emissions to the North Sea Environment. Int. J. Appl. Sci. SOFW-Journal, 141 (3), 40-46

Grand View Research, Inc. (2016). Traffic Road Marking Coating Market Analysis By Product (Paint, Thermoplastic, Preformed Polymer Tape, Epoxy) And Segment - Forecasts To 2022.

GRPE (2013). Particulate Matter Emissions by Tyres.

Jambeck, J.R., Geyer, R., Wilcox, C., Siegler, T.R., Perryman, M., Andrady, A., Narayan, R., and Law, K.L. (2015). Plastic waste inputs from land into the ocean. Science 347, 768771.

https://doi.org/10.1126/science.1260352

Laaksonen, T. (2012). A market analysis on the global boating industry (Tampere University of applied sciences), Bachelor Thesis, 36pp,

Lassen, C., Foss Hansen, S., Magnusson, K., Noren, F., Bloch Hartmann, N.I., Rehne Jensen, P., Gisel Nielsen, T., and Brinch, A. (2015). Microplastics: Occurrence, effects and sources of releases to the environment in Denmark (The Danish Environmental Protection Agency).

Lechner, A., Keckeis, H., Lumesberger-Loisl, F., Zens, B., Krusch, R., Tritthart, M., Glas, M., and Schludermann, E. (2014). The Danube so colourful: A potpourri of plastic litter outnumbers fish larvae in Europe's second largest river. Environ. Pollut. 188, 177-181.

https://doi.org/10.1016/j.envpol.2014.02.006

Leslie, H.A. (2015). Plastic in Cosmetics: are we polluting the environment through our personal care? UNEP 2015, $33 \mathrm{pp}$.

Macfadyen, G., Huntington, T., and Cappel, R. (2009). Abandoned, lost or otherwise discarded fishing gear (FAO and UNEP). FAO Fisheries and Aquaculture Technical paper 523. UNEP Regional Seas Reports and Studies 185. $115 \mathrm{pp}$

Magnuson, K., Eliason, K., Frane, A., Haikonen, K., Hulten, J., Olshammar, M., Stadmark, J., and Voisin, A. (2016). Swedish sources and pathways for microplastics to the marine environment. www.ivl.se/webdav/files/Rapporter/C183.pdf

McKinsey\&Company, and Ocean Conservancy (2015). Stemming the tide : Land-based strategies for a plastic-free ocean. 
OECD (2013). Environment at a Glance 2013. OECD indicators.

https://doi.org/10.1787/9789264185715-en

OECD Series on emissions documents (2009). Emission Scenarion documents on coating industry (Paints, Laquers and Varnishes).

Pakula, C., and Stamminger, R. (2010). Electricity and water consumption for laundry washing by washing machine worldwide. Energy Effic. 3, 365-382

https://doi.org/10.1007/s12053-009-9072-8

Phillips, P.J., Chalmers, A.T., Gray, J.L., Kolpin, D.W., Foreman, W.T., and Wall, G.R. (2012). Combined Sewer Overflows: An Environmental Source of Hormones and Wastewater Micropollutants. Environ. Sci. Technol. 46, 5336-5343. https://doi.org/10.1021/es3001294

PlasticsEurope (2009). The compelling facts about plastics 2009 - an analysis of European plastics production demand ans recovery for 2008.

PlasticsEurope (2015). Plastics - The facts 2015. An analysis of European plastics production, demand and waste data analysis of European plastics production, demand and waste data.

Raynaud, J. (2014). Valuing Plastic: the business case for measuring, managing and disclosing plastic use in the consumer goods industry (UNEP).

Reed, C. (2016). Plastic Age: How it's reshaping rocks, oceans and life. NewScientist, Feature 28 January 2015

RIVM (2014). Quick scan and Prioritization of Microplastic Sources and Emissions (National Institute for Public Health and the Environment (RIVM)). Report 2014-0156, 48pp.

Sebille, E. van, Wilcox, C., Lebreton, L., Maximenko, N., Hardesty, B.D., Franeker, J.A. van, Eriksen, M., Siegel, D., Galgani, F., and Law, K.L. (2015). A global inventory of small floating plastic debris. Environ. Res. Lett. 10, 124006. https://doi.org/10.1088/1748-9326/10/12/124006

Seltenrich, N. (2015). New Link in the Food Chain? Marine Plastic Pollution and Seafood Safety. Environ. Health Perspect. 123, A34-A41.

https://doi.org/10.1289/ehp.123-a34

Sundt, P., Schulze, P.-E., and Syversen, F. (2014). Sources of microplastic- pollution to the marine environment (MEPEX). wWw.miljodirektoratet.no/Documents/publikasjoner/M321/ M321.pdf

Sundt, P., Syversen, F., Skogesal, O., and Schulze, P.-E. (2016). Primary microplastic-pollution: Measures and reduction potentials in Norway (MEPEX). www.miljodirektoratet.no/ Documents/publikasjoner/M545/M545.pdf

Ten Broeke, H., Hulskotte, J., and Denier van der Gon, H. (2008). Road traffic tyre wear.http://www.emissieregistratie. nl/ERPUBLIEK/documenten/Water/Factsheets/English/ Road\%20traffic\%20tyre\%20wear.pdf

Thevenon, F., Caroll, C., and Sousa, J. (2014). Plastic Debris in the Oceans. (IUCN). The Characterization of Marine Plastics and their Environmental Impacts, Situation Analysis
Report. Gland, Switzerland: IUCN. 52 pp.

Tukker, A., Bulavskaya T., and Giljum S., de Koning A., Lutter S., Simas M., Stadler K., Wood R. (2014). The Global Resource Footprint of Nations. Carbon, water, land and materials embodied in trade and final consumption calculated with EXIOBASE 2.1. (Leiden/Delft/Vienna/ Trondheim: Organisation for Applied Scientific Research / Vienna University of Economics and Business / Norwegian University of Science and Technology).

UNDP (2015). World Population Prospects: The 2015 Revision. (New York: United Nations, Department of Economic and Social Affairs, Population Division).

UNEP SETAC (2009). Life Cycle Management - how business uses it to decrease footprint, create opportunities and make value chains more sustainable.

United Nations Human Settlements Programme (2016). Urbanization and development: emerging futures. World cities report 2016

UNSD (Division de statistique de l'ONU, 2011). UNSD Environmental Indicators: Wastewater. Wastewater. http:// www.unstats.un.org/unsd/environment/wastewater.htm

Wang, R., Balkanski, Y., Boucher, O., Bopp, L., Chappell, A., Ciais, P., Hauglustaine, D., Penuelas, J., and Tao, S. (2015) Sources, transport and deposition of iron in the global atmosphere. Atmospheric Chem. Phys. 6247-6270. https://doi.org/10.5194/acp-15-6247-2015

Woodall, L.C., Sanchez-Vidal, A., Canals, M., Paterson, G.L.J., Coppock, R., Sleight, V., Calafat, A., Rogers, A.D., Narayanaswamy, B.E., and Thompson, R.C. (2014). The deep sea is a major sink for microplastic debris. Open Sci. 1, 140317

https://doi.org/10.1098/rsos.140317

World Economic Forum, Ellen MacArthur Foundation, and McKinsey\&Company (2016). The new plastic econom - rethinking the future of plastics. http://www. ellenmacarthurfoundation.org/publications/the-newplastics-economy-rethinking-the- future-of-plastics.

WRAP (2006). The Composition of a Tyre: Typical Components. http://www.wrap.org.uk/sites/files/wrap/2\% 20-\%20Composition\%20 of\%20a\%20Tyre\%20-\%20May\% 202006.pdf

Wright, T. (2009). Marine Coating Market. Rodman Media. 


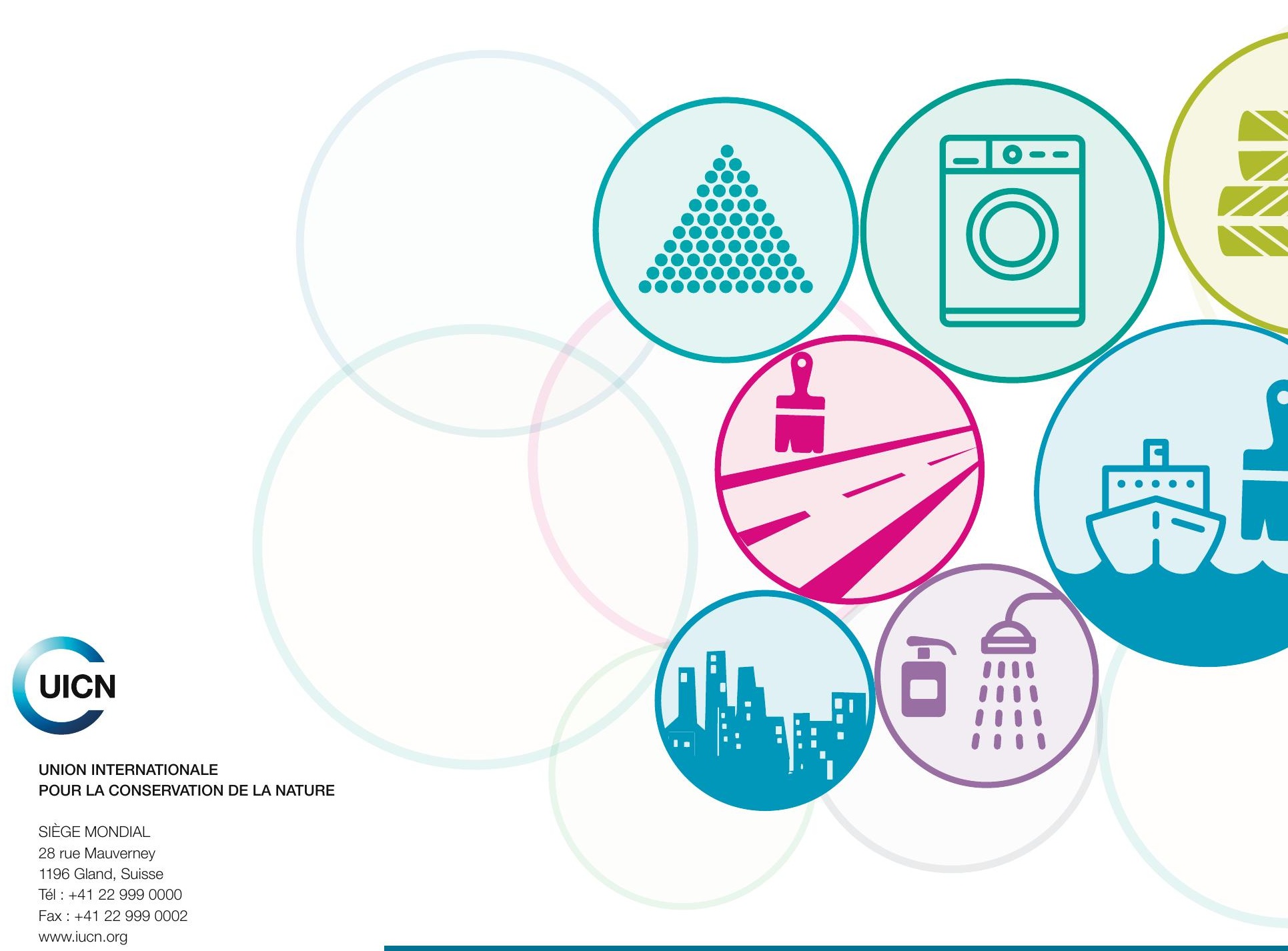

